\title{
MULTIPLE POINTS OF OPERATOR SEMISTABLE LÉVY PROCESSES
}

\author{
TOMASZ LUKS AND YIMIN XIAO
}

\begin{abstract}
We determine the Hausdorff dimension of the set of $k$-multiple points for a symmetric operator semistable Lévy process $X=\left\{X(t), t \in \mathbb{R}_{+}\right\}$in terms of the eigenvalues of its stability exponent. We also give a necessary and sufficient condition for the existence of $k$-multiple points. Our results extend to all $k \geq 2$ the recent work [23, where the set of double points $(k=2)$ was studied in the symmetric operator stable case.
\end{abstract}

\section{IntRoduction AND STATEMENT OF THE RESULTS}

The questions on the existence of multiple points (or intersections) and the Hausdorff dimension of the set of multiple points of stochastic processes have been of considerable interest for many years. The problem was originally studied for Brownian motion by Dvoretzky, Erdös, Kakutani and Taylor [4, 5, 6. Their results were later extended to more general Lévy processes using various techniques, see [7, 8, 9, 11, 12, 15, 18, 19, 21, 23, 26, 28, 29, 30 and the references therein. Let $X=\left\{X(t), t \in \mathbb{R}_{+}\right\}$be a stochastic process with values in $\mathbb{R}^{d}, d \geq 1$, and let $k \geq 2$ be an integer. A point $x \in \mathbb{R}^{d}$ is called a $k$-multiple point of $X$ if there exist $k$ distinct times $t_{1}, \ldots, t_{k} \in \mathbb{R}_{+}$such that

$$
X\left(t_{1}\right)=\ldots=X\left(t_{k}\right)=x .
$$

If $k=2$, then $x$ is also called a double point of $X$. We denote by $M_{k}$ the set of $k$ multiple points of $X$. The objective of this paper is to study the set $M_{k}$ for symmetric operator semistable Lévy processes and to extend to all $k \geq 2$ the recent results of [23, where the set of double points was investigated when $X$ is symmetric operator stable. Recall from [25, 27] that a Lévy process $X$ is called operator semistable if the distribution $\nu$ of $X(1)$ is full (i.e. not supported on any lower dimensional hyperplane) and there exists a linear operator $B$ on $\mathbb{R}^{d}$ such that

$$
\nu^{c}=c^{B} \nu \quad \text { for some } c>1,
$$

where $\nu^{c}$ denotes the $c$-fold convolution power of the infinitely divisible law $\nu$ and $c^{B} \nu(d x)=\nu\left(c^{-B} d x\right)$ is the image measure of $\nu$ under the linear operator $c^{B}$. The operator $B$ is called a stability exponent of $X$. We refer to [3, 13, 20, 22, and to the monograph [25] for more comprehensive information on operator semistable laws. As

2010 Mathematics Subject Classification. 60J25, 60J30, 60G51, 60G17.

Key words and phrases. Multiple points, Hausdorff dimension, Operator semistable process, Lévy process.

Research of Y. Xiao was partially supported by the NSF Grants DMS-1612885 and DMS-1607089. 
a consequence of (11), an operator semistable Lévy process $X$ is also operator semiselfsimilar, i.e., for the constant $c>1$ in (1),

$$
\{X(c t)\}_{t \geq 0} \stackrel{\mathrm{fd}}{=}\left\{c^{B} X(t)\right\}_{t \geq 0},
$$

where $\stackrel{\mathrm{fd}}{=}$ denotes equality of all finite-dimensional distributions of the process. Operator semi-selfsimilar processes constitute a much broader class than that of semi-selfsimilar processes, see [24] for more details. If (11) (resp. (22)) holds for all $c>0$, the Lévy process $X$ is called operator stable (resp. operator self-similar).

To formulate our main results, we factor the minimal polynomial of the stability exponent $B$ into $q_{1}(x) \cdots q_{p}(x)$, where all roots of $q_{i}(x)$ have real parts $a_{i}$ and $a_{i}<a_{j}$ for $i<j$. Define $V_{i}=\operatorname{Ker}\left(q_{i}(B)\right)$ and $d_{i}=\operatorname{dim}\left(V_{i}\right)$. Then $d_{1}+\cdots+d_{p}=d$ and $V_{1} \oplus \cdots \oplus V_{p}$ is a direct sum decomposition of $\mathbb{R}^{d}$ into $B$-invariant subspaces. We may write $B=B_{1} \oplus \cdots \oplus B_{p}$, where $B_{i}: V_{i} \rightarrow V_{i}$ and every eigenvalue of $B_{i}$ has real part equal to $a_{i}$. For $j=1, \ldots, d$ and $l=1, \ldots, p$, denote $\alpha_{j}=a_{l}^{-1}$ whenever $\sum_{i=0}^{l-1} d_{i}<j \leq \sum_{i=0}^{l} d_{i}$, where $d_{0}:=0$. We then have $\alpha_{1} \geq \ldots \geq \alpha_{d}$, and note that $0<\alpha_{j} \leq 2$ in view of [25, Theorem 7.2.1].

Our first theorem provides an explicit formula for the Hausdorff dimension of $M_{k}$ in $\mathbb{R}^{2}$ in terms of the indices $\alpha_{j}$.

Theorem 1. Let $X=\left\{X(t), t \in \mathbb{R}_{+}\right\}$be a symmetric operator semistable Lévy process in $\mathbb{R}^{2}$ with exponent $B$ and let $M_{k}$ be the set of $k$-multiple points of $X$. Then for all $k \geq 2$ we have almost surely

$$
\operatorname{dim}_{\mathrm{H}} M_{k}=\min \left\{\alpha_{1}\left(k-(k-1)\left(\alpha_{1}^{-1}+\alpha_{2}^{-1}\right)\right), 2-k \alpha_{2}\left(\alpha_{1}^{-1}+\alpha_{2}^{-1}-1\right)\right\},
$$

where a negative dimension means that $M_{k}=\emptyset$ almost surely.

Theorem 1 is more general than [28, Theorem 1], where $B$ is assumed to be a diagonal matrix with entries on the diagonal $\alpha_{j} \in(1,2)(1 \leq j \leq d)$. Note also that the dimension formula for double points in $\mathbb{R}^{2}$ and $\mathbb{R}^{3}$ is given in [14, Corollary 3.8]. Since $M_{3}=\emptyset$ a.s. for $d=3$ and $M_{2}=\emptyset$ a.s. for $d \geq 4$ (see the beginning of Section 4 for the proof), Theorem 1 completes the solution of the Hausdorff dimension problem for $M_{k}$ in the setting of symmetric operator semistable Lévy processes.

Our second theorem characterizes the existence of multiple points in terms of $\alpha_{1}$ and $\alpha_{2}$. According to the Jordan decomposition for $d=2$, the stability exponent of $X$ satisfies $B=P D P^{-1}$ for some real invertible matrix $P$ and a matrix $D$ which can have the following forms:

$$
\begin{aligned}
& \text { (A.1) }\left(\begin{array}{cc}
1 / \alpha_{1} & 0 \\
0 & 1 / \alpha_{2}
\end{array}\right) \text { or }\left(\begin{array}{cc}
1 / \alpha_{1} & -b \\
b & 1 / \alpha_{2}
\end{array}\right) \text { with } \alpha_{1}=\alpha_{2} \text {; } \\
& \text { (A.2) }\left(\begin{array}{cc}
1 / \alpha & 0 \\
1 & 1 / \alpha
\end{array}\right) .
\end{aligned}
$$

Clearly, in the case (A.2) we have $\alpha:=\alpha_{1}=\alpha_{2}$.

Theorem 2. Let $X=\left\{X(t), t \in \mathbb{R}_{+}\right\}$be a symmetric operator semistable Lévy process in $\mathbb{R}^{d}$ and let $k \geq 3$. The existence of $k$-multiple points of $X$ for $d=2$ depends on the cases (A.1) and (A.2) as follows: 
- In Case (A.1), $M_{k} \neq \emptyset$ a.s. if and only if $k-(k-1)\left(\alpha_{1}^{-1}+\alpha_{2}^{-1}\right)>0$.

- In Case (A.2), $M_{k} \neq \emptyset$ a.s. if and only if $\alpha \geq 2(k-1) / k$.

Furthermore, $M_{k}=\emptyset$ a.s. for $d \geq 3$.

The existence of double points is characterized separately in Corollary 6. Note that in the case (A.2) and $k \geq 3$, the set $M_{k}$ is nonempty almost surely if $\alpha=2(k-1) / k$ although its Hausdorff dimension is zero. The same effect appears for double points in $\mathbb{R}^{3}$ in the non-diagonalizable case (B.3) and $\alpha=3 / 2$ (see the end of Section 4 for details).

Even though in this paper we focus on symmetric operator semistable Lévy process in $\mathbb{R}^{d}$ with $d \geq 2$ so that its exponent $B$ is a matrix, the problems on existence of $k$-multiple points and the Hausdorff dimension of $M_{k}$ are meaningful for the case of $d=1$ as well. In the special case when $X=\left\{X(t), t \in \mathbb{R}_{+}\right\}$is a symmetric stable Lévy process in $\mathbb{R}$, these problems were solved by Taylor [29]. For a symmetric semistable Lévy process in $\mathbb{R}$, we can apply the general tools in [16, 17, 21, 23] (cf. (12) and (3) below) to solve these problems. We provide more details in Remark 8 .

This paper raises several natural questions on multiple points of operator semistable Lévy processes. For example, it would be interesting to remove the "symmetry" assumption; or to find an exact Hausdorff measure function for $M_{k}$. Another interesting problem is to find the packing dimension of $M_{k}$.

Our paper is organized as follows. In Section 2 we give basic definitions and recall some useful facts. In Section 3 we prove Theorem 1. In Section 4 we deal with the existence problem for multiple points and prove Theorem 2 .

\section{Preliminaries}

A stochastic process $X=\left\{X(t), t \in \mathbb{R}_{+}\right\}$with values in $\mathbb{R}^{d}$ is called a Lévy process if $X$ has stationary and independent increments, $X(0)=0$ a.s. and $t \mapsto X(t)$ is continuous in probability. We refer to the books [1, 27 for systematic accounts on Lévy processes. It is known that the finite-dimensional distributions of $X$ are determined by the characteristic function

$$
\mathbb{E}\left[e^{i\langle\xi, X(t)\rangle}\right]=e^{-t \Psi(\xi)}, \quad \forall t \geq 0,
$$

where $\Psi: \mathbb{R}^{d} \mapsto \mathbb{C}$ is given by the Lévy-Khintchine formula and is called the characteristic or Lévy exponent of $X$.

A Lévy process $X$ is said to be symmetric if $-X$ and $X$ have the same finitedimensional distributions. In such a case, $\Psi(\xi) \geq 0$ for all $\xi \in \mathbb{R}^{d}$. Using the terminology in [16, 17], we say that $X$ is absolutely continuous, if for all $t>0$, the function $\xi \mapsto e^{-t \Psi(\xi)}$ is in $L^{1}\left(\mathbb{R}^{d}\right)$. In this case, the Fourier inversion formula implies that the density function of $X(t)$ is bounded and continuous.

It has been recently proved in [23, Theorem 1] that the Hausdorff dimension of $M_{k}$ for a symmetric, absolutely continuous Lévy process with characteristic exponent $\Psi$ is given by

$$
\operatorname{dim}_{\mathrm{H}} M_{k}=d-\inf \left\{\beta \in(0, d]: \int_{\mathbb{R}^{k d}}\left[\frac{1}{1+\left\|\sum_{l=1}^{k} \xi_{l}\right\|^{\beta}} \prod_{j=1}^{k} \frac{1}{1+\Psi\left(\xi_{j}\right)}\right] d \bar{\xi}<\infty\right\}
$$


almost surely, where $\bar{\xi}=\left(\xi_{1}, \ldots, \xi_{k}\right)$ for $\xi_{j} \in \mathbb{R}^{d}$ and $\|\cdot\|$ denotes the usual Euclidean norm in $\mathbb{R}^{d}$. Here we use the convention $\inf \emptyset=d$.

Furthermore, in the case when $X$ is a symmetric operator semistable Lévy process, [14, Corollary 2.2] gives the following estimate for its characteristic exponent $\Psi$ : for every $\varepsilon>0$, there exists a constant $\tau>1$ such that for all $\xi \in \mathbb{R}^{d}$ with $\|\xi\| \geq \tau$, we have

$$
\frac{K^{-1}}{\|\xi\|^{\varepsilon} \sum_{j=1}^{d}\left|\xi_{j}\right|^{\alpha_{j}}} \leq \frac{1}{1+\Psi(\xi)} \leq \frac{K}{\sum_{j=1}^{d}\left|\xi_{j}\right|^{\alpha_{j}}}
$$

where $K \geq 1$ is a constant which depends on $\varepsilon$ and $\tau$ only and, as defined in the Introduction, $\alpha_{1} \geq \ldots \geq \alpha_{d}>0$ are the reciprocals of the real parts of the eigenvalues of the stability exponent $B$. The estimate (4) extends [26, Theorem 4.2], where the result was obtained for operator stable Lévy processes, and implies that $X$ is absolutely continuous.

Throughout the rest of the paper, $C$ will denote a positive constant, whose value may change in each appearance and depends on the coefficients $\alpha_{j}$ and the multiplicity $k$ only. By the notation $f \asymp g$ we mean that there is a constant $C$ such that $C^{-1} g \leq$ $f \leq C g$. Analogously we define $\gtrsim$ and $\lesssim$.

\section{HausdorfF Dimension OF MUltiple points in $\mathbb{R}^{2}$}

Our goal in this section is to prove Theorem 1 by extending the method of [23] in order to deal with the set $M_{k}$ for $k \geq 3$. To this end we fix $2 \geq \alpha_{1} \geq \alpha_{2}>0$ and let $X=\left\{X(t), t \in \mathbb{R}_{+}\right\}$be a symmetric operator semistable Lévy process in $\mathbb{R}^{2}$ with

stability exponent $B$ whose eigenvalues have real parts $\alpha_{1}^{-1}, \alpha_{2}^{-1}$, as explained in the Introduction. Denote

$$
A_{k}:=\left\{\left(x_{1}, \ldots, x_{k}\right) \in \mathbb{R}^{2 k}:\left\|x_{i}\right\|>1, i=1, \ldots, k\right\},
$$

where $x_{i}=\left(x_{i 1}, x_{i 2}\right) \in \mathbb{R}^{2}$ for $i=1, \ldots, k$, and for $\beta>0$ let

$$
I_{\beta}:=\int \cdots \int_{A_{k}} \frac{1}{1+\left|\sum_{i=1}^{k} x_{i 1}\right|^{\beta}+\left|\sum_{i=1}^{k} x_{i 2}\right|^{\beta}} \prod_{i=1}^{k} \frac{d x_{1} \ldots d x_{k}}{\left|x_{i 1}\right|^{\alpha_{1}}+\left|x_{i 2}\right|^{\alpha_{2}}} .
$$

It follows from (3), (44) and a slight modification of [23, Lemma 2] that

$$
\operatorname{dim}_{\mathrm{H}} M_{k}=2-\inf \left\{\beta \in(0,2): I_{\beta}<\infty\right\}
$$

almost surely. For $q, r \geq 1$ let

$$
\begin{aligned}
& A_{k}(q, r):=\left\{\left(x_{1}, \ldots, x_{k}\right)\right. \in \mathbb{R}^{2 k}:\left\|x_{i}\right\|>1, i=1, \ldots, k, \\
&\left.q-1 \leq\left|\sum_{i=1}^{k} x_{i 1}\right|<q, r-1 \leq\left|\sum_{i=1}^{k} x_{i 2}\right|<r\right\} .
\end{aligned}
$$

We have

(6) $I_{\beta}<\infty$ if and only if $\sum_{m, n \in \mathbb{N}} \frac{1}{m^{\beta}+n^{\beta}} \int \cdots \int_{A_{k}(m, n)} \prod_{i=1}^{k} \frac{d x_{1} \ldots d x_{k}}{\left|x_{i 1}\right|^{\alpha_{1}}+\left|x_{i 2}\right|^{\alpha_{2}}}<\infty$. 
In [23, Proof of Theorem 4] it has been shown that, excepting the cases $\alpha_{1}=\alpha_{2}=2$ and $\alpha_{1}=2, \alpha_{2}=1$, one has

$$
\iint_{A_{2}(m, n)} \frac{d x_{1} d x_{2}}{\left(\left|x_{11}\right|^{\alpha_{1}}+\left|x_{12}\right|^{\alpha_{2}}\right)\left(\left|x_{21}\right|^{\alpha_{1}}+\left|x_{22}\right|^{\alpha_{2}}\right)} \asymp\left(m^{\alpha_{1}}+n^{\alpha_{2}}\right)^{1 / \alpha_{1}+1 / \alpha_{2}-2}
$$

for any $m, n \in \mathbb{N}$ provided $2-1 / \alpha_{1}-1 / \alpha_{2}>0$ and the integral above is infinite otherwise. When $\alpha_{1}=\alpha_{2}=2$ or $\alpha_{1}=2$ and $\alpha_{2}=1$, an extra factor $\log m$ or $\log n$ may appear in the upper bound of (7), the rest of the statement remains the same. Our next result extends (7) to all $k \geq 2$, which is essential for proving Theorem 1,

Proposition 3. Assume $\alpha_{2}<2$ and let $k \geq 2$ be an integer. For $k=2=\alpha_{1}$ assume in addition $\alpha_{2} \neq 1$. Then for any real numbers $q, r \geq 1$ we have

$$
\int \ldots \int_{A_{k}(q, r)} \prod_{i=1}^{k} \frac{1}{\left|x_{i 1}\right|^{\alpha_{1}}+\left|x_{i 2}\right|^{\alpha_{2}}} d x_{1} \ldots d x_{k} \asymp\left(q^{\alpha_{1}}+r^{\alpha_{2}}\right)^{(k-1)\left(1 / \alpha_{1}+1 / \alpha_{2}\right)-k}
$$

provided $k-(k-1)\left(1 / \alpha_{1}+1 / \alpha_{2}\right)>0$, and the integral is infinite otherwise.

Proof. Notice that the integrand in (8) is symmetric in $x_{i 1}$ and $x_{i 2}$ for $i=1, \ldots, k$. Hence, without loss of generality, we will consider the integral in (8) over the set

$$
\begin{gathered}
\widetilde{A}_{k}(q, r):=\left\{\left(x_{1}, \ldots, x_{k}\right) \in \mathbb{R}^{2 k}: x_{k 1}, x_{k 2} \geq 1,\left|x_{i 1}\right|,\left|x_{i 2}\right| \geq 1, i=1, \ldots, k-1,\right. \\
\left.q-1 \leq\left|\sum_{i=1}^{k} x_{i 1}\right|<q, r-1 \leq\left|\sum_{i=1}^{k} x_{i 2}\right|<r\right\} .
\end{gathered}
$$

We then have $q-1 \leq\left|\sum_{i=1}^{k} x_{i 1}\right|<q$ if and only if

$$
-q-x_{k 1} \leq \sum_{i=1}^{k-1} x_{i 1} \leq-q-x_{k 1}+1 \quad \text { or } \quad q-x_{k 1}-1 \leq \sum_{i=1}^{k-1} x_{i 1} \leq q-x_{k 1}
$$

and $r-1 \leq\left|\sum_{i=1}^{k} x_{i 2}\right|<r$ if and only if

$$
-r-x_{k 2} \leq \sum_{i=1}^{k-1} x_{i 2} \leq-r-x_{k 2}+1 \quad \text { or } \quad r-x_{k 2}-1 \leq \sum_{i=1}^{k-1} x_{i 2} \leq r-x_{k 2} .
$$

Consider the following four cases:

1). $q+x_{k 1}-1 \leq\left|\sum_{i=1}^{k-1} x_{i 1}\right| \leq q+x_{k 1}$ and $x_{k 1} \geq 1$.

2). $q-x_{k 1}-1 \leq\left|\sum_{i=1}^{k-1} x_{i 1}\right| \leq q-x_{k 1}$ and $1 \leq x_{k 1} \leq q-1$.

3). $\left|\sum_{i=1}^{k-1} x_{i 1}\right| \leq 2$ and $q-1 \leq x_{k 1} \leq q+1$.

4). $x_{k 1}-q \leq\left|\sum_{i=1}^{k-1} x_{i 1}\right| \leq x_{k 1}-q+1$ and $x_{k 1} \geq q+1$.

It can be seen that the condition $q-1 \leq\left|\sum_{i=1}^{k} x_{i 1}\right|<q$ implies one of the four cases above. Consider analogous cases implied by $r-1 \leq\left|\sum_{i=1}^{k} x_{i 2}\right|<r$, and for $i, j=1,2,3,4$ let $A_{k}^{i, j}(q, r)$ denote the subset of

$$
\left\{\left(x_{1}, \ldots, x_{k}\right) \in \mathbb{R}^{2 k}: x_{k 1}, x_{k 2} \geq 1,\left|x_{i 1}\right|,\left|x_{i 2}\right| \geq 1, i=1, \ldots, k-1\right\}
$$


with the case $i$ applied to $x_{11}, \ldots, x_{k 1}$ and the case $j$ applied to $x_{12}, \ldots, x_{k 2}$. For instance,

$$
\begin{aligned}
A_{k}^{1,1}(q, r) & =\left\{\left(x_{1}, \ldots, x_{k}\right) \in \mathbb{R}^{2 k}: x_{k 1}, x_{k 2} \geq 1,\left|x_{i 1}\right|,\left|x_{i 2}\right| \geq 1, i=1, \ldots, k-1\right. \\
q & \left.+x_{k 1}-1 \leq\left|\sum_{i=1}^{k-1} x_{i 1}\right| \leq q+x_{k 1}, \quad r+x_{k 2}-1 \leq\left|\sum_{i=1}^{k-1} x_{i 2}\right|<r+x_{k 2}\right\} .
\end{aligned}
$$

We have

$$
A_{k}^{1,1}(q, r) \subseteq \widetilde{A}_{k}(q, r) \subseteq \bigcup_{i, j=1}^{4} A_{k}^{i, j}(q, r) .
$$

Hence, it is enough to show the lower bound of (8) with the integration restricted to $A_{k}^{1,1}(q, r)$ and the upper bound of (8) with the integration over all $A_{k}^{i, j}(q, r)$.

We proceed by induction on $k$. Note that the proposition is proved to hold for $k=2$ except the upper bound in the case $\alpha_{1}=2$ and $\alpha_{2}=1$. Clearly, analyzing [23, Proof of Theorem 4] one can easily deduce that (7) holds also for any non-integer numbers $m, n \geq 1$.

Assume now the statement of the proposition holds for some $k \geq 2$ and let $k^{\prime}=k+1$. Consider first the integration over $A_{k^{\prime}}^{1,1}(q, r)$. By applying the induction hypothesis to $A_{k}\left(q+x_{k^{\prime} 1}, r+x_{k^{\prime} 2}\right)$ we get

$$
\begin{aligned}
& \int \cdots \int_{A_{k^{\prime}}^{1,1}(q, r)} \prod_{i=1}^{k^{\prime}} \frac{1}{\left|x_{i 1}\right|^{\alpha_{1}}+\left|x_{i 2}\right|^{\alpha_{2}}} d x_{1} \ldots d x_{k^{\prime}} \\
& \asymp \int_{1}^{\infty} \int_{1}^{\infty}\left(\frac{1}{\left(q+x_{k^{\prime} 1}\right)^{\alpha_{1}}+\left(r+x_{k^{\prime}}\right)^{\alpha_{2}}}\right)^{k-(k-1)\left(1 / \alpha_{1}+1 / \alpha_{2}\right)} \frac{d x_{k^{\prime} 1} d x_{k^{\prime} 2}}{x_{k^{\prime} 1}^{\alpha_{1}}+x_{k^{\prime} 2}^{\alpha_{2}}} \\
& \asymp \int_{1}^{\infty} \int_{1}^{\infty}\left(\frac{1}{q^{\alpha_{1}}+r^{\alpha_{2}}+t^{\alpha_{1}}+s^{\alpha_{2}}}\right)^{k-(k-1)\left(1 / \alpha_{1}+1 / \alpha_{2}\right)} \frac{d t d s}{t^{\alpha_{1}}+s^{\alpha_{2}}} \\
& \asymp \int_{1}^{\infty} \int_{1}^{\infty}\left(\frac{1}{q^{\alpha_{1}}+r^{\alpha_{2}}} \wedge \frac{1}{t^{\alpha_{1}}} \wedge \frac{1}{s^{\alpha_{2}}}\right)^{k-(k-1)\left(1 / \alpha_{1}+1 / \alpha_{2}\right)}\left(\frac{1}{t^{\alpha_{1}}} \wedge \frac{1}{s^{\alpha_{2}}}\right) d t d s \\
& =\int_{1}^{\infty} \int_{1}^{s^{\alpha_{2} / \alpha_{1}}} \frac{1}{s^{\alpha_{2}}}\left(\frac{1}{q^{\alpha_{1}}+r^{\alpha_{2}}} \wedge \frac{1}{s^{\alpha_{2}}}\right)^{k-(k-1)\left(1 / \alpha_{1}+1 / \alpha_{2}\right)} d t d s \\
& \quad+\int_{1}^{\infty} \int_{s^{\alpha_{2} / \alpha_{1}}}^{\infty} \frac{1}{t^{\alpha_{1}}}\left(\frac{1}{q^{\alpha_{1}}+r^{\alpha_{2}}} \wedge \frac{1}{t^{\alpha_{1}}}\right)^{k-(k-1)\left(1 / \alpha_{1}+1 / \alpha_{2}\right)} d t d s \\
& :=I_{1}+I_{2} .
\end{aligned}
$$


Recall that for $k=2=\alpha_{1}$ and $\alpha_{2}=1$ only the lower bound of the above is true. We have

$$
\begin{aligned}
I_{2}= & \int_{1}^{\infty} \int_{s^{\alpha_{2} / \alpha_{1}}}^{\infty} \frac{1}{t^{\alpha_{1}}}\left(\frac{1}{q^{\alpha_{1}}+r^{\alpha_{2}}} \wedge \frac{1}{t^{\alpha_{1}}}\right)^{k-(k-1)\left(1 / \alpha_{1}+1 / \alpha_{2}\right)} d t d s \\
= & \int_{1}^{\left(q^{\alpha_{1}}+r^{\alpha_{2}}\right)^{1 / \alpha_{2}}} \int_{s^{\alpha_{2} / \alpha_{1}}}^{\infty} \frac{1}{t^{\alpha_{1}}}\left(\frac{1}{q^{\alpha_{1}}+r^{\alpha_{2}}} \wedge \frac{1}{t^{\alpha_{1}}}\right)^{k-(k-1)\left(1 / \alpha_{1}+1 / \alpha_{2}\right)} d t d s \\
& +\int_{\left(q^{\alpha_{1}}+r^{\alpha_{2}}\right)^{1 / \alpha_{2}}}^{\infty} \int_{s^{\alpha_{2} / \alpha_{1}}}^{\infty} t^{(k-1)\left(1+\alpha_{1} / \alpha_{2}\right)-k^{\prime} \alpha_{1}} d t d s:=I_{2}^{(1)}+I_{2}^{(2)} .
\end{aligned}
$$

For $k^{\prime}-k\left(1 / \alpha_{1}+1 / \alpha_{2}\right)>0$ we get

$$
I_{2}^{(2)}=C \int_{\left(q^{\left.\alpha_{1}+r^{\alpha_{2}}\right)^{1 / \alpha_{2}}}\right.}^{\infty} s^{k\left(\alpha_{2} / \alpha_{1}+1\right)-k^{\prime} \alpha_{2}-1} d s=C\left(q^{\alpha_{1}}+r^{\alpha_{2}}\right)^{k\left(1 / \alpha_{1}+1 / \alpha_{2}\right)-k^{\prime}} .
$$

This gives the lower bound in (8) . If $k^{\prime}-k\left(1 / \alpha_{1}+1 / \alpha_{2}\right) \leq 0$, then $I_{2}^{(2)}=\infty$, so the same holds for the integral in (8) and the last part of the statement follows.

It remains to prove the upper bound in (8) for the case of $k^{\prime}-k\left(1 / \alpha_{1}+1 / \alpha_{2}\right)>0$. Note that the latter also implies $\alpha_{2}>1$, so the restriction in the case $k=2$ is no longer needed. We now proceed to bound the integrals in (8) over the sets $A_{k^{\prime}}^{i, j}(q, r)$, separately.

On the set $A_{k^{\prime}}^{1,1}(q, r)$, we make use of (9) and (10) and derive

$$
\begin{aligned}
& I_{2}^{(1)}=\int_{1}^{\left(q^{\alpha_{1}}+r^{\alpha_{2}}\right)^{1 / \alpha_{2}}} \int_{s^{\alpha_{2} / \alpha_{1}}}^{\infty} \frac{1}{t^{\alpha_{1}}}\left(\frac{1}{q^{\alpha_{1}}+r^{\alpha_{2}}} \wedge \frac{1}{t^{\alpha_{1}}}\right)^{k-(k-1)\left(1 / \alpha_{1}+1 / \alpha_{2}\right)} d t d s \\
& =\left(\frac{1}{q^{\alpha_{1}}+r^{\alpha_{2}}}\right)^{k-(k-1)\left(1 / \alpha_{1}+1 / \alpha_{2}\right)} \int_{1}^{\left(q^{\alpha_{1}}+r^{\alpha_{2}}\right)^{1 / \alpha_{2}}} \int_{s^{\alpha_{2} / \alpha_{1}}}^{\left(q^{\alpha_{1}}+r^{\alpha_{2}}\right)^{1 / \alpha_{1}}} t^{-\alpha_{1}} d t d s \\
& +\int_{1}^{\left(q^{\alpha_{1}}+r^{\alpha_{2}}\right)^{1 / \alpha_{2}}} \int_{\left(q^{\alpha_{1}}+r^{\alpha_{2}}\right)^{1 / \alpha_{1}}}^{\infty} t^{(k-1)\left(1+\alpha_{1} / \alpha_{2}\right)-k^{\prime} \alpha_{1}} d t d s \\
& \lesssim\left(\frac{1}{q^{\alpha_{1}}+r^{\alpha_{2}}}\right)^{k-(k-1)\left(1 / \alpha_{1}+1 / \alpha_{2}\right)} \int_{1}^{\left(q^{\alpha_{1}}+r^{\alpha_{2}}\right)^{1 / \alpha_{2}}} s^{\alpha_{2} / \alpha_{1}-\alpha_{2}} d s \\
& +\int_{1}^{\left(q^{\alpha_{1}}+r^{\alpha_{2}}\right)^{1 / \alpha_{2}}}\left(q^{\alpha_{1}}+r^{\alpha_{2}}\right)^{(k-1)\left(1 / \alpha_{1}+1 / \alpha_{2}\right)-k^{\prime}+1 / \alpha_{1}} d s \\
& \lesssim\left(q^{\alpha_{1}}+r^{\alpha_{2}}\right)^{k\left(1 / \alpha_{1}+1 / \alpha_{2}\right)-k^{\prime}},
\end{aligned}
$$


where the last inequality follows from the assumption $\alpha_{2}<2$. Furthermore,

$$
\begin{aligned}
I_{1} & =\int_{1}^{\infty} \int_{1}^{s^{\alpha_{2} / \alpha_{1}}} \frac{1}{s^{\alpha_{2}}}\left(\frac{1}{q^{\alpha_{1}}+r^{\alpha_{2}}} \wedge \frac{1}{s^{\alpha_{2}}}\right)^{k-(k-1)\left(1 / \alpha_{1}+1 / \alpha_{2}\right)} d t d s \\
& \leq \int_{1}^{\infty} s^{\alpha_{2} / \alpha_{1}-\alpha_{2}}\left(\frac{1}{q^{\alpha_{1}}+r^{\alpha_{2}}} \wedge \frac{1}{s^{\alpha_{2}}}\right)^{k-(k-1)\left(1 / \alpha_{1}+1 / \alpha_{2}\right)} d s \\
& =\left(\frac{1}{q^{\alpha_{1}}+r^{\alpha_{2}}}\right)^{k-(k-1)\left(1 / \alpha_{1}+1 / \alpha_{2}\right)} \int_{1}^{\left(q^{\left.\alpha_{1}+r^{\alpha_{2}}\right)^{1 / \alpha_{2}}} s^{\alpha_{2} / \alpha_{1}-\alpha_{2}} d s\right.} \\
& +\int_{\left(q^{\alpha_{1}}+r^{\alpha_{2}}\right)^{1 / \alpha_{2}}}^{\infty} s^{k\left(\alpha_{2} / \alpha_{1}+1\right)-k^{\prime} \alpha_{2}-1} d s \\
& \lesssim\left(q^{\alpha_{1}}+r^{\alpha_{2}}\right)^{k\left(1 / \alpha_{1}+1 / \alpha_{2}\right)-k^{\prime}} .
\end{aligned}
$$

This proves the desired upper bound for the integral in (8) over $A_{k^{\prime}}^{1,1}(q, r)$.

Next we consider the integral over $A_{k^{\prime}}^{2,2}(q, r)$. By applying the induction hypothesis to the integral over $A_{k}\left(q-x_{k^{\prime} 1}, r-x_{k^{\prime} 2}\right)$ we get

$$
\begin{aligned}
& \int \cdots \int_{A_{k^{\prime}(q, r)}^{2,2}} \prod_{i=1}^{k^{\prime}} \frac{1}{\left|x_{i 1}\right|^{\alpha_{1}}+\left|x_{i 2}\right|^{\alpha_{2}}} d x_{1} \ldots d x_{k^{\prime}} \\
& \asymp \int_{1}^{r-1} \int_{1}^{q-1}\left(\frac{1}{\left(q-x_{k^{\prime} 1}\right)^{\alpha_{1}}+\left(r-x_{k^{\prime} 2}\right)^{\alpha_{2}}}\right)^{k-(k-1)\left(1 / \alpha_{1}+1 / \alpha_{2}\right)} \frac{d x_{k^{\prime} 1} d x_{k^{\prime} 2}}{x_{k^{\prime} 1}^{\alpha_{1}}+x_{k^{\prime} 2}^{\alpha_{2}}} \\
& \leq\left(q^{\alpha_{1}}+r^{\alpha_{2}}\right)^{(k-1)\left(1 / \alpha_{1}+1 / \alpha_{2}-1\right)} \int_{1}^{r-1} \int_{1}^{q-1} \frac{d t d s}{\left((q-t)^{\alpha_{1}}+(r-s)^{\alpha_{2}}\right)\left(t^{\alpha_{1}}+s^{\alpha_{2}}\right)} .
\end{aligned}
$$

The last double integral above has already appeared in [23, (11)], so it follows from [23, Proof of Theorem 4] that

$$
\int_{1}^{r-1} \int_{1}^{q-1} \frac{d t d s}{\left((q-t)^{\alpha_{1}}+(r-s)^{\alpha_{2}}\right)\left(t^{\alpha_{1}}+s^{\alpha_{2}}\right)} \leq C\left(q^{\alpha_{1}}+r^{\alpha_{2}}\right)^{1 / \alpha_{1}+1 / \alpha_{2}-2} .
$$

This gives the desired upper bound for the integral over $A_{k^{\prime}}^{2,2}(q, r)$.

For the integral over $A_{k^{\prime}}^{3,3}(q, r)$, we have

$$
\begin{aligned}
& \int \cdots \int_{A_{k^{\prime}(q, r)}^{3,3}} \prod_{i=1}^{k^{\prime}} \frac{1}{\left|x_{i 1}\right|^{\alpha_{1}}+\left|x_{i 2}\right|^{\alpha_{2}}} d x_{1} \ldots d x_{k^{\prime}} \\
& \lesssim \frac{1}{q^{\alpha_{1}}+r^{\alpha_{2}}} \int \cdots \int_{B_{k}} \prod_{i=1}^{k} \frac{1}{\left|x_{i 1}\right|^{\alpha_{1}}+\left|x_{i 2}\right|^{\alpha_{2}}} d x_{1} \ldots d x_{k}
\end{aligned}
$$

where $B_{k}=A_{k}(1,1) \cup A_{k}(2,2) \cup A_{k}(2,1) \cup A_{k}(1,2)$. By the induction hypothesis, the last multiple integral is finite provided $k-(k-1)\left(1 / \alpha_{1}+1 / \alpha_{2}\right)>0$. Since $1 / \alpha_{1}+1 / \alpha_{2} \geq 1$, we have

$$
k-(k-1)\left(1 / \alpha_{1}+1 / \alpha_{2}\right) \geq k^{\prime}-k\left(1 / \alpha_{1}+1 / \alpha_{2}\right)>0 .
$$

Furthermore, $\left(q^{\alpha_{1}}+r^{\alpha_{2}}\right)^{-1} \leq\left(q^{\alpha_{1}}+r^{\alpha_{2}}\right)^{k\left(1 / \alpha_{1}+1 / \alpha_{2}\right)-k^{\prime}}$. This gives the upper bound in (8) with the integration over $A_{k^{\prime}}^{3,3}(q, r)$. 
Consider $A_{k^{\prime}}^{4,4}(q, r)$. We apply the induction hypothesis to the integration over $A_{k}\left(x_{k^{\prime} 1}-q, x_{k^{\prime} 2}-r\right)$ and get

$$
\begin{aligned}
& \int \cdots \int_{A_{k^{\prime}}^{4,4}(q, r)} \prod_{i=1}^{k^{\prime}} \frac{1}{\left|x_{i 1}\right|^{\alpha_{1}}+\left|x_{i 2}\right|^{\alpha_{2}}} d x_{1} \ldots d x_{k^{\prime}} \\
& \asymp \int_{r+1}^{\infty} \int_{q+1}^{\infty}\left(\frac{1}{\left(x_{k^{\prime} 1}-q\right)^{\alpha_{1}}+\left(x_{k^{\prime} 2}-r\right)^{\alpha_{2}}}\right)^{k-(k-1)\left(1 / \alpha_{1}+1 / \alpha_{2}\right)} \frac{d x_{k^{\prime} 1} d x_{k^{\prime} 2}}{x_{k^{\prime} 1}^{\alpha_{1}}+x_{k^{\prime} 2}^{\alpha_{2}}} .
\end{aligned}
$$

After the change of variables $t=x_{k^{\prime} 1}-q$ and $s=x_{k^{\prime} 2}-r$ the last term equals

$$
\begin{aligned}
& \int_{1}^{\infty} \int_{1}^{\infty}\left(\frac{1}{t^{\alpha_{1}}+s^{\alpha_{2}}}\right)^{k-(k-1)\left(1 / \alpha_{1}+1 / \alpha_{2}\right)} \frac{d t d s}{(t+q)^{\alpha_{1}}+(s+r)^{\alpha_{2}}} \\
& \asymp \int_{1}^{\infty} \int_{1}^{\infty}\left(\frac{1}{t^{\alpha_{1}}+s^{\alpha_{2}}}\right)^{k-(k-1)\left(1 / \alpha_{1}+1 / \alpha_{2}\right)} \frac{d t d s}{q^{\alpha_{1}}+r^{\alpha_{2}}+t^{\alpha_{1}}+s^{\alpha_{2}}} \\
& \asymp \int_{1}^{\infty} \int_{1}^{\infty}\left(\frac{1}{t^{\alpha_{1}}} \wedge \frac{1}{s^{\alpha_{2}}}\right)^{k-(k-1)\left(1 / \alpha_{1}+1 / \alpha_{2}\right)}\left(\frac{1}{q^{\alpha_{1}}+r^{\alpha_{2}}} \wedge \frac{1}{t^{\alpha_{1}}} \wedge \frac{1}{s^{\alpha_{2}}}\right) d t d s \\
& =\int_{1}^{\infty} \int_{1}^{s^{\alpha_{2} / \alpha_{1}}} s^{(k-1)\left(1+\alpha_{2} / \alpha_{1}\right)-k \alpha_{2}}\left(\frac{1}{q^{\alpha_{1}}+r^{\alpha_{2}}} \wedge \frac{1}{s^{\alpha_{2}}}\right) d t d s \\
& +\int_{1}^{\infty} \int_{s^{\alpha_{2} / \alpha_{1}}}^{\infty} t^{(k-1)\left(1+\alpha_{1} / \alpha_{2}\right)-k \alpha_{1}}\left(\frac{1}{q^{\alpha_{1}}+r^{\alpha_{2}}} \wedge \frac{1}{t^{\alpha_{1}}}\right) d t d s=\widetilde{I}_{1}+\widetilde{I}_{2} .
\end{aligned}
$$

We have

$$
\begin{aligned}
\widetilde{I}_{1} & \leq \int_{1}^{\infty} s^{k\left(1+\alpha_{2} / \alpha_{1}-\alpha_{2}\right)-1}\left(\frac{1}{q^{\alpha_{1}}+r^{\alpha_{2}}} \wedge \frac{1}{s^{\alpha_{2}}}\right) d s \\
& =\frac{1}{q^{\alpha_{1}}+r^{\alpha_{2}}} \int_{1}^{\left(q^{\alpha_{1}}+r^{\alpha_{2}}\right)^{1 / \alpha_{2}}} s^{k\left(1+\alpha_{2} / \alpha_{1}-\alpha_{2}\right)-1} d s+\int_{\left(q^{\left.\alpha_{1}+r^{\alpha_{2}}\right)^{1 / \alpha_{2}}}\right.}^{\infty} s^{k\left(1+\alpha_{2} / \alpha_{1}\right)-k^{\prime} \alpha_{2}-1} d s \\
& \lesssim\left(q^{\alpha_{1}}+r^{\alpha_{2}}\right)^{k\left(1 / \alpha_{1}+1 / \alpha_{2}\right)-k^{\prime}} .
\end{aligned}
$$

In the last step above we have used again the assumption $\alpha_{2}<2$. Furthermore,

$$
\begin{aligned}
& \widetilde{I}_{2}=\frac{1}{q^{\alpha_{1}}+r^{\alpha_{2}}} \int_{1}^{\left(q^{\left.\alpha_{1}+r^{\alpha_{2}}\right)^{1 / \alpha_{2}}}\right.} \int_{s^{\alpha_{2} / \alpha_{1}}}^{\left(q^{\left.\alpha_{1}+r^{\alpha_{2}}\right)^{1 / \alpha_{1}}}\right.} t^{(k-1)\left(1+\alpha_{1} / \alpha_{2}\right)-k \alpha_{1}} d t d s
\end{aligned}
$$

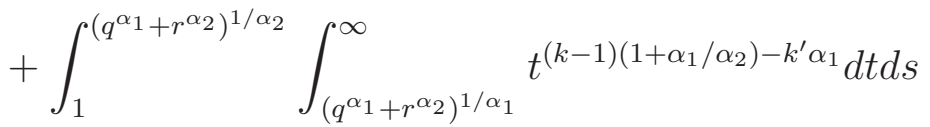

$$
\begin{aligned}
& +\int_{\left(q^{\alpha_{1}}+r^{\alpha_{2}}\right)^{1 / \alpha_{2}}}^{\infty} \int_{s^{\alpha_{2} / \alpha_{1}}}^{\infty} t^{(k-1)\left(1+\alpha_{1} / \alpha_{2}\right)-k^{\prime} \alpha_{1}} d t d s .
\end{aligned}
$$

The last two integrals appeared already in the case of $A_{k^{\prime}}^{1,1}(q, r)$, i.e., $I_{2}^{(2)}$ and the second part of $I_{2}^{(1)}$, thus they are less than $C\left(q^{\alpha_{1}}+r^{\alpha_{2}}\right)^{k\left(1 / \alpha_{1}+1 / \alpha_{2}\right)-k^{\prime}}$. In order to estimate the 
remaining term we note again that $1 / \alpha_{1}+1 / \alpha_{2} \geq 1$, hence

$$
\begin{aligned}
& \frac{1}{q^{\alpha_{1}}+r^{\alpha_{2}}} \int_{1}^{\left(q^{\alpha_{1}}+r^{\alpha_{2}}\right)^{1 / \alpha_{2}}} \int_{s^{\alpha_{2} / \alpha_{1}}}^{\left(q^{\alpha_{1}}+r^{\alpha_{2}}\right)^{1 / \alpha_{1}}} t^{-\alpha_{1}}\left(t^{\alpha_{1}}\right)^{(k-1)\left(1 / \alpha_{1}+1 / \alpha_{2}-1\right)} d t d s \\
& \leq\left(q^{\alpha_{1}}+r^{\alpha_{2}}\right)^{(k-1)\left(1 / \alpha_{1}+1 / \alpha_{2}\right)-k} \int_{1}^{\left(q^{\alpha_{1}}+r^{\alpha_{2}}\right)^{1 / \alpha_{2}}} \int_{s^{\alpha_{2} / \alpha_{1}}}^{\left(q^{\alpha_{1}}+r^{\alpha_{2}}\right)^{1 / \alpha_{1}}} t^{-\alpha_{1}} d t d s .
\end{aligned}
$$

Since the last term is equal to the first part of $I_{2}^{(1)}$, the desired upper bound follows.

Consider the integration over $A_{k^{\prime}}^{1,2}(q, r)$. Again, by applying the induction hypotheses to $A_{k}\left(q+x_{k^{\prime} 1}, r-x_{k^{\prime} 2}\right)$, we get

$$
\begin{aligned}
& \int \cdots \int_{A_{k^{\prime}(q, r)}^{1,2}} \prod_{i=1}^{k^{\prime}} \frac{1}{\left|x_{i 1}\right|^{\alpha_{1}}+\left|x_{i 2}\right|^{\alpha_{2}}} d x_{1} \ldots d x_{k^{\prime}} \\
& \asymp \int_{1}^{r-1} \int_{1}^{\infty}\left(\frac{1}{\left(q+x_{k^{\prime} 1}\right)^{\alpha_{1}}+\left(r-x_{k^{\prime} 2}\right)^{\alpha_{2}}}\right)^{k-(k-1)\left(1 / \alpha_{1}+1 / \alpha_{2}\right)} \frac{d x_{k^{\prime} 1} d x_{k^{\prime} 2}}{x_{k^{\prime} 1}^{\alpha_{1}}+x_{k^{\prime} 2}^{\alpha_{2}}} \\
& \lesssim \int_{1}^{\infty}(q+t)^{(k-1)\left(1+\alpha_{1} / \alpha_{2}\right)-k \alpha_{1}} \int_{1}^{r-1}\left(s+t^{\alpha_{1} / \alpha_{2}}\right)^{-\alpha_{2}} d s d t \\
& =\int_{1}^{\infty}(q+t)^{(k-1)\left(1+\alpha_{1} / \alpha_{2}\right)-k \alpha_{1}} \int_{1+t^{\alpha_{1} / \alpha_{2}}}^{r+t^{\alpha_{1} / \alpha_{2}-1}} s^{-\alpha_{2}} d s d t .
\end{aligned}
$$

Recall that the condition $k^{\prime}-k\left(1 / \alpha_{1}+1 / \alpha_{2}\right)>0$ implies $\alpha_{2}>1$, hence the last integral is less than

$$
\begin{aligned}
& C \int_{1}^{\infty}(q+t)^{(k-1)\left(1+\alpha_{1} / \alpha_{2}\right)-k \alpha_{1}} t^{\alpha_{1} / \alpha_{2}-\alpha_{1}} d t \\
& \lesssim q^{(k-1)\left(1+\alpha_{1} / \alpha_{2}\right)-k \alpha_{1}} \int_{1}^{q} t^{\alpha_{1} / \alpha_{2}-\alpha_{1}} d t+\int_{q}^{\infty} t^{k\left(1+\alpha_{1} / \alpha_{2}\right)-k^{\prime} \alpha_{1}-1} d t \\
& \lesssim q^{k\left(1+\alpha_{1} / \alpha_{2}\right)-k^{\prime} \alpha_{1}} .
\end{aligned}
$$

It remains to estimate the integral (11) in terms of $r$. We have

$$
\begin{aligned}
& \int_{1}^{r / 2} \int_{1}^{\infty}\left(\frac{1}{(q+t)^{\alpha_{1}}+(r-s)^{\alpha_{2}}}\right)^{k-(k-1)\left(1 / \alpha_{1}+1 / \alpha_{2}\right)} \frac{d t d s}{t^{\alpha_{1}}+s^{\alpha_{2}}} \\
& \lesssim r^{(k-1)\left(1+\alpha_{2} / \alpha_{1}\right)-k \alpha_{2}} \int_{1}^{r / 2} \int_{1}^{\infty}\left(t+s^{\alpha_{2} / \alpha_{1}}\right)^{-\alpha_{1}} d t d s \\
& \lesssim r^{(k-1)\left(1+\alpha_{2} / \alpha_{1}\right)-k \alpha_{2}} \int_{1}^{r / 2} s^{\alpha_{2} / \alpha_{1}-\alpha_{2}} d s .
\end{aligned}
$$

Since $\alpha_{2}<2$, we have $\alpha_{2} / \alpha_{1}-\alpha_{2}>-1$ and the last term above is less than $C r^{k\left(1+\alpha_{2} / \alpha_{1}\right)-k^{\prime} \alpha_{2}}$. 
Furthermore, for the second part of (111) one gets

$$
\begin{aligned}
& \int_{r / 2}^{r-1} \int_{1}^{\infty}\left(\frac{1}{(q+t)^{\alpha_{1}}+(r-s)^{\alpha_{2}}}\right)^{k-(k-1)\left(1 / \alpha_{1}+1 / \alpha_{2}\right)} \frac{d t d s}{t^{\alpha_{1}}+s^{\alpha_{2}}} \\
& =\int_{1}^{r / 2} \int_{1}^{\infty-(k-1)\left(1 / \alpha_{1}+1 / \alpha_{2}\right)} \frac{d t d u}{t^{\alpha_{1}}+(r-u)^{\alpha_{2}}} \\
& \lesssim \int_{1}^{r / 2} \int_{1}^{\infty}\left(\frac{1}{t^{\alpha_{1}}+u^{\alpha_{2}}}\right)^{k-(k-1)\left(1 / \alpha_{1}+1 / \alpha_{2}\right)} \frac{d t d u}{t^{\alpha_{1}}+r^{\alpha_{2}}} \\
& =\int_{1}^{r / 2} \int_{1}^{\infty} \frac{\left(t^{\alpha_{1}}+u^{\alpha_{2}}\right)^{(k-1)\left(1 / \alpha_{1}+1 / \alpha_{2}-1\right)}}{t^{\alpha_{1}}+u^{\alpha_{2}}} \frac{d t d u}{t^{\alpha_{1}}+r^{\alpha_{2}}} \\
& \leq \int_{1}^{r / 2} \int_{1}^{\infty}\left(\frac{1}{t^{\alpha_{1}}+r^{\alpha_{2}}}\right)^{k-(k-1)\left(1 / \alpha_{1}+1 / \alpha_{2}\right)} \frac{d t d u}{t^{\alpha_{1}}+u^{\alpha_{2}}} \\
& \lesssim r^{(k-1)\left(1+\alpha_{2} / \alpha_{1}\right)-k \alpha_{2}} \int_{1}^{r / 2} \int_{1}^{\infty}\left(t+u^{\alpha_{2} / \alpha_{1}}\right)^{-\alpha_{1}} d t d u \\
& \lesssim r^{k\left(1+\alpha_{2} / \alpha_{1}\right)-k^{\prime} \alpha_{2}},
\end{aligned}
$$

where the last inequality follows from estimating the first part of (11). Altogether we get that (11) is less than

$$
C\left(q^{k\left(1+\alpha_{1} / \alpha_{2}\right)-k^{\prime} \alpha_{1}} \wedge r^{k\left(1+\alpha_{2} / \alpha_{1}\right)-k^{\prime} \alpha_{2}}\right) \asymp\left(q^{\alpha_{1}}+r^{\alpha_{2}}\right)^{k\left(1 / \alpha_{1}+1 / \alpha_{2}\right)-k^{\prime}} .
$$

The case of $A_{k^{\prime}}^{2,1}(q, r)$ is similar. Consider the integration over $A_{k^{\prime}}^{2,4}(q, r)$. The induction hypothesis applied to $A_{k}\left(q-x_{k^{\prime} 1}, x_{k^{\prime} 2}-r\right)$ gives

$$
\begin{aligned}
& \int \cdots \int_{A_{k^{\prime}, 4}(q, r)} \prod_{i=1}^{k^{\prime}} \frac{1}{\left|x_{i 1}\right|^{\alpha_{1}}+\left|x_{i 2}\right|^{\alpha_{2}}} d x_{1} \ldots d x_{k^{\prime}} \\
& \asymp \int_{r+1}^{\infty} \int_{1}^{q-1}\left(\frac{1}{(q-t)^{\alpha_{1}}+(s-r)^{\alpha_{2}}}\right)^{k-(k-1)\left(1 / \alpha_{1}+1 / \alpha_{2}\right)} \frac{d t d s}{t^{\alpha_{1}}+s^{\alpha_{2}}} \\
& =\int_{1}^{\infty} \int_{1}^{q-(k-1)\left(1 / \alpha_{1}+1 / \alpha_{2}\right)} \frac{d t d u}{t^{\alpha_{1}}+(u+r)^{\alpha_{2}}} \\
& =\int_{1}^{r-1} \int_{1}^{q-1} \ldots+\int_{r-1}^{\infty} \int_{1}^{q-1} \ldots=\widehat{I}_{1}+\widehat{I}_{2} .
\end{aligned}
$$

In the last equality we have assumed $r \geq 2$, the case $1 \leq r<2$ being irrelevant. We have

$$
\begin{aligned}
\widehat{I_{1}} & \leq \int_{1}^{r-1} \int_{1}^{q-1}\left(\frac{1}{(q-t)^{\alpha_{1}}+u^{\alpha_{2}}}\right)^{k-(k-1)\left(1 / \alpha_{1}+1 / \alpha_{2}\right)} \frac{d t d u}{t^{\alpha_{1}}+(r-u)^{\alpha_{2}}} \\
& =\int_{1}^{r-1} \int_{1}^{q-1}\left(\frac{1}{(q-t)^{\alpha_{1}}+(r-v)^{\alpha_{2}}}\right)^{k-(k-1)\left(1 / \alpha_{1}+1 / \alpha_{2}\right)} \frac{d t d v}{\frac{t^{\alpha_{1}}+v^{\alpha_{2}}}{}}
\end{aligned}
$$


Note that the integral above is exactly the one from the case of $A_{k^{\prime}}^{2,2}(q, r)$, so it is less than $C\left(q^{\alpha_{1}}+r^{\alpha_{2}}\right)^{k\left(1 / \alpha_{1}+1 / \alpha_{2}\right)-k^{\prime}}$. Furthermore,

$$
\begin{aligned}
\widehat{I}_{2} & =\int_{1}^{\infty} \int_{1}^{q-1}\left(\frac{1}{(q-t)^{\alpha_{1}}+(v+r-2)^{\alpha_{2}}}\right)^{k-(k-1)\left(1 / \alpha_{1}+1 / \alpha_{2}\right)} \frac{d t d v}{t^{\alpha_{1}}+(v+2 r-2)^{\alpha_{2}}} \\
& \lesssim \int_{1}^{\infty} \int_{1}^{q-1}\left(\frac{1}{(q-t)^{\alpha_{1}}+(v+r)^{\alpha_{2}}}\right)^{k-(k-1)\left(1 / \alpha_{1}+1 / \alpha_{2}\right)} \frac{d t d v}{\frac{t^{\alpha_{1}}+v^{\alpha_{2}}}{}}
\end{aligned}
$$

The last integral above appears in the case of $A_{k^{\prime}}^{2,1}(q, r)$, so it is less than $C\left(q^{\alpha_{1}}+\right.$ $\left.r^{\alpha_{2}}\right)^{k\left(1 / \alpha_{1}+1 / \alpha_{2}\right)-k^{\prime}}$. This gives the upper bound with the integration over $A_{k^{\prime}}^{2,4}(q, r)$. The proof for $A_{k^{\prime}}^{4,2}(q, r)$ is similar.

It remains to show the upper bound of (8) with the integration over the sets $A_{k^{\prime}}^{1,3}(q, r)$, $A_{k^{\prime}}^{3,1}(q, r), A_{k^{\prime}}^{2,3}(q, r), A_{k^{\prime}}^{3,2}(q, r), A_{k^{\prime}}^{3,4}(q, r)$ and $A_{k^{\prime}}^{4,3}(q, r)$. This can be done in a similar way and is actually easier since the integration is reduced to a smaller number of variables. We omit the details.

In order to evaluate the right hand side of (5), we prove the following equality which extends [23, Lemma 1].

Lemma 4. We have

$$
\begin{aligned}
& \inf \left\{\beta \in(0,2]: \sum_{m=1}^{\infty} \sum_{n=1}^{\infty} \frac{1}{m^{\beta}+n^{\beta}}\left(\frac{1}{m^{\alpha_{1}}+n^{\alpha_{2}}}\right)^{k-(k-1)\left(1 / \alpha_{1}+1 / \alpha_{2}\right)}<\infty\right\} \\
& =\max \left\{2-\alpha_{1}\left(k-(k-1)\left(\alpha_{1}^{-1}+\alpha_{2}^{-1}\right)\right), k \alpha_{2}\left(\alpha_{1}^{-1}+\alpha_{2}^{-1}-1\right)\right\} .
\end{aligned}
$$

Proof. The convergence of the series is equivalent to the convergence of the integral

$$
\begin{aligned}
& \int_{1}^{\infty} \int_{1}^{\infty}\left(\frac{1}{x} \wedge \frac{1}{y}\right)^{\beta}\left(\frac{1}{x^{\alpha_{1}}} \wedge \frac{1}{y^{\alpha_{2}}}\right)^{k-(k-1)\left(1 / \alpha_{1}+1 / \alpha_{2}\right)} d x d y \\
& =\int_{1}^{\infty} \int_{1}^{y^{\alpha_{2} / \alpha_{1}}} \frac{1}{y^{\beta}}\left(\frac{1}{y^{\alpha_{2}}}\right)^{k-(k-1)\left(1 / \alpha_{1}+1 / \alpha_{2}\right)} d x d y \\
& \quad+\int_{1}^{\infty} \int_{y^{\alpha_{2} / \alpha_{1}}}^{y} \frac{1}{y^{\beta}}\left(\frac{1}{x^{\alpha_{1}}}\right)^{k-(k-1)\left(1 / \alpha_{1}+1 / \alpha_{2}\right)} d x d y \\
& \quad+\int_{1}^{\infty} \int_{y}^{\infty} \frac{1}{x^{\beta}}\left(\frac{1}{x^{\alpha_{1}}}\right)^{k-(k-1)\left(1 / \alpha_{1}+1 / \alpha_{2}\right)} d y \\
& :=I_{1}+I_{2}+I_{3} .
\end{aligned}
$$

It can be seen that $I_{1}<\infty$ if and only if

$$
\int_{1}^{\infty} y^{k \alpha_{2}\left(1 / \alpha_{1}+1 / \alpha_{2}-1\right)-\beta-1} d y<\infty
$$

and the last condition is equivalent with $\beta>k \alpha_{2}\left(\alpha_{1}^{-1}+\alpha_{2}^{-1}-1\right)$. 
Next we consider $I_{2}$. If $\alpha_{1}=\alpha_{2}$, then $I_{2}=0$, so assume that $\alpha_{1} \neq \alpha_{2}$. If $-k \alpha_{1}+$ $(k-1)\left(1+\alpha_{1} / \alpha_{2}\right)=-1$, then

$$
I_{2}=\left(1-\alpha_{2} / \alpha_{1}\right) \int_{1}^{\infty} y^{-\beta} \ln y d y
$$

So $I_{2}<\infty$ if and only if $\beta>1=2-\alpha_{1}\left(k-(k-1)\left(\alpha_{1}^{-1}+\alpha_{2}^{-1}\right)\right)$. Suppose now $-k \alpha_{1}+(k-1)\left(1+\alpha_{1} / \alpha_{2}\right) \neq-1$. Then we have

$$
I_{2}=\int_{1}^{\infty} y^{-\beta}\left(\frac{y^{1-k \alpha_{1}+(k-1)\left(1+\alpha_{1} / \alpha_{2}\right)}-\left(y^{\alpha_{2} / \alpha_{1}}\right)^{1-k \alpha_{1}+(k-1)\left(1+\alpha_{1} / \alpha_{2}\right)}}{1-k \alpha_{1}+(k-1)\left(1+\alpha_{1} / \alpha_{2}\right)}\right) d y .
$$

We consider two cases:

(a) If $1-k \alpha_{1}+(k-1)\left(1+\alpha_{1} / \alpha_{2}\right)>0$, then

$$
I_{2} \leq C \int_{1}^{\infty} y^{1-k \alpha_{1}+(k-1)\left(1+\alpha_{1} / \alpha_{2}\right)-\beta} d y
$$

and the last integral is finite if $\beta>2-\alpha_{1}\left(k-(k-1)\left(\alpha_{1}^{-1}+\alpha_{2}^{-1}\right)\right)$.

(b) If $1-k \alpha_{1}+(k-1)\left(1+\alpha_{1} / \alpha_{2}\right)<0$, then

$$
I_{2} \leq C \int_{1}^{\infty} y^{k \alpha_{2}\left(1 / \alpha_{1}+1 / \alpha_{2}-1\right)-1-\beta} d y
$$

which is finite if $\beta>k \alpha_{2}\left(\alpha_{1}^{-1}+\alpha_{2}^{-1}-1\right)$.

Therefore, the condition

$$
\beta>\max \left\{2-\alpha_{1}\left(k-(k-1)\left(\alpha_{1}^{-1}+\alpha_{2}^{-1}\right)\right), k \alpha_{2}\left(\alpha_{1}^{-1}+\alpha_{2}^{-1}-1\right)\right\}
$$

implies $I_{2}<\infty$.

Finally, we consider $I_{3}$. A necessary condition for $I_{3}<\infty$ is

$$
-k \alpha_{1}+(k-1)\left(1+\alpha_{1} / \alpha_{2}\right)-\beta<-1 \text {. }
$$

Assuming this we get

$$
I_{3}=\int_{1}^{\infty} \frac{y^{1-k \alpha_{1}+(k-1)\left(1+\alpha_{1} / \alpha_{2}\right)-\beta}}{1-k \alpha_{1}+(k-1)\left(1+\alpha_{1} / \alpha_{2}\right)-\beta} d y .
$$

Thus $I_{3}<\infty$ if and only if $\beta>2-\alpha_{1}\left(k-(k-1)\left(\alpha_{1}^{-1}+\alpha_{2}^{-1}\right)\right)$.

Therefore, we have proved that the condition

$$
\beta>\max \left\{2-\alpha_{1}\left(k-(k-1)\left(\alpha_{1}^{-1}+\alpha_{2}^{-1}\right)\right), k \alpha_{2}\left(\alpha_{1}^{-1}+\alpha_{2}^{-1}-1\right)\right\}
$$

implies $I_{1}, I_{2}, I_{3}<\infty$, and that the condition $I_{1}, I_{3}<\infty$ implies

$$
\beta>\max \left\{2-\alpha_{1}\left(k-(k-1)\left(\alpha_{1}^{-1}+\alpha_{2}^{-1}\right)\right), k \alpha_{2}\left(\alpha_{1}^{-1}+\alpha_{2}^{-1}-1\right)\right\} .
$$

This yields the conclusion of the lemma.

Proof of Theorem 11. For $k=2$, the theorem is a reformulation of [14, Corollary 3.8, Part (a)]. For $k \geq 3$ and $\alpha_{2}<2$, the statement is a direct consequence of (5) , (6) ), Proposition 3 and Lemma 4. When $\alpha_{2}=\alpha_{1}=2$, we may apply Proposition 3 with $\widetilde{\alpha}_{2}:=\alpha_{2}-\varepsilon$ and let $\varepsilon \rightarrow 0$ in order to obtain an upper bound for $\beta$ in (5). However, this upper bound turns out to be 0 , so the proof is complete. 


\section{Existence of $k$-MUltiple POints With $k \geq 3$}

We will now focus on proving Theorem 2, Since $X$ is symmetric, its continuous transition density satisfies

$$
p_{t}(0)=\int_{\mathbb{R}^{d}}\left(p_{t / 2}(x)\right)^{2} d x>0 .
$$

It follows from [21, Proof of Theorem 1] that the existence of $k$-multiple points of $X$ is equivalent to the existence of intersections of $k$ independent copies of $X$. Furthermore, by [17, Theorem 2.1], $X$ is weakly unimodal. Hence, by [16, Remark 6.6], $k$ independent copies of $X$ intersect if and only if

$$
\int_{\mathbb{R}^{d(k-1)}} \frac{1}{1+\Psi\left(\sum_{j=1}^{k-1} x_{j}\right)} \prod_{j=1}^{k-1} \frac{1}{1+\Psi\left(x_{j}\right)} d \bar{x}<\infty .
$$

We refer to [8, 21, 10, 19] for appropriate conditions for the existence of intersections in terms of the potential density. Consider first $d=k=3$. Making the change of variables $x_{1}=\xi_{1}-\xi_{2}, x_{2}=\xi_{2}$ in (12), we conclude that $M_{3}=\emptyset$ a.s. if and only if

$$
\int_{\mathbb{R}^{3}} \int_{\mathbb{R}^{3}} \frac{1}{1+\Psi\left(\xi_{1}\right)} \cdot \frac{1}{1+\Psi\left(\xi_{1}-\xi_{2}\right)} \cdot \frac{1}{1+\Psi\left(\xi_{2}\right)} d \xi_{1} d \xi_{2}<\infty .
$$

Since $\Psi(\xi) \leq C\|\xi\|^{2}$ for all $\xi \in \mathbb{R}^{3}$ with $\|\xi\|$ large enough, there exists $N \in \mathbb{N}$ such that the integral in (13) can be estimated from below by

$$
C \sum_{i=N}^{\infty} \frac{1}{i^{2}} \iint_{\|x\|,\|y\| \geq N} \frac{1}{\|x\|^{2}\|y\|^{2}} \mathbb{1}_{\{i-1 \leq\|x-y\|<i\}} d x d y .
$$

Note that $\int \mathbb{1}_{\{i-1 \leq\|x-y\|<i\}} d y \asymp i^{2}$, hence the last term above is greater than

$$
C \sum_{i=N}^{\infty} \int_{\|x\| \geq N} \frac{1}{\|x\|^{2}} \cdot \frac{1}{\|x\|^{2}+i^{2}} d x \gtrsim \sum_{i=N}^{\infty} \int_{\|x\| \geq i}\|x\|^{-4} d x \asymp \sum_{i=N}^{\infty} \frac{1}{i}=\infty .
$$

This shows that $M_{3}=\emptyset$ a.s. for $d=3$ and the last statement of Theorem 2 follows. Furthermore, when $k=2$, (12) is equivalent to

$$
\int_{\mathbb{R}^{d}}\left(\frac{1}{1+\Psi(\xi)}\right)^{2} d \xi<\infty
$$

Applying the same estimate of $\Psi$ as before we conclude that $M_{2}=\emptyset$ a.s. for $d \geq 4$.

Recall from the Introduction that for $d=2$ the stability exponent of $X$ satisfies $B=P D P^{-1}$, where the matrix $D$ can have the following forms

(A.1) $\left(\begin{array}{cc}1 / \alpha_{1} & 0 \\ 0 & 1 / \alpha_{2}\end{array}\right)$ or $\left(\begin{array}{cc}1 / \alpha_{1} & -b \\ b & 1 / \alpha_{2}\end{array}\right)$ with $\alpha_{1}=\alpha_{2}$;

(A.2) $\left(\begin{array}{cc}1 / \alpha & 0 \\ 1 & 1 / \alpha\end{array}\right)$.

We split the proof of Theorem 2 into two parts, according to the cases (A.1) and (A.2). 
Proof of Theorem 2, Case (A.1). Note that, by Theorem 1, the Hausdorff dimension of $M_{k}$ is strictly positive for $\alpha_{1}=\alpha_{2}=2$. Hence, without loss of generality we may assume $\alpha_{2}<2$. By [14, (2.1),(2.5),(2.6),(2.7)] we have

$$
\Psi(x) \asymp\left|x_{1}\right|^{\alpha_{1}}+\left|x_{2}\right|^{\alpha_{2}} \quad \text { for }\|x\| \geq C .
$$

This implies that condition (12) is equivalent to

$$
\int_{\mathbb{R}^{2(k-1)}} \frac{1}{1+\left|\sum_{j=1}^{k-1} x_{j 1}\right|^{\alpha_{1}}+\left|\sum_{j=1}^{k-1} x_{j 2}\right|^{\alpha_{2}}} \prod_{j=1}^{k-1} \frac{1}{1+\left|x_{j 1}\right|^{\alpha_{1}}+\left|x_{j 2}\right|^{\alpha_{2}}} d \bar{x}<\infty .
$$

The last integral is finite if and only if

$$
\sum_{m, n \in \mathbb{N}} \frac{1}{m^{\alpha_{1}}+n^{\alpha_{2}}} \int \cdots \int_{A_{k-1}(m, n)} \prod_{i=1}^{k-1} \frac{1}{\left|x_{i 1}\right|^{\alpha_{1}}+\left|x_{i 2}\right|^{\alpha_{2}}} d x_{1} \ldots d x_{k-1}<\infty
$$

where the sets $A_{k-1}(m, n)$ were defined at the beginning of Section 3 . Finally, by Proposition 3, the last condition is equivalent to

$$
\sum_{m, n \in \mathbb{N}}\left(\frac{1}{m^{\alpha_{1}}+n^{\alpha_{2}}}\right)^{k-(k-2)\left(1 / \alpha_{1}+1 / \alpha_{2}\right)}<\infty,
$$

and it is quite straightforward to see, using similar methods as in Lemma 4, that the last series is convergent if and only if $k-(k-1)\left(\alpha_{1}^{-1}+\alpha_{2}^{-1}\right)>0$.

Observe that, since $\alpha_{2} \leq \alpha_{1}$, the second term in the dimension formula of Theorem 1 satisfies

$$
\begin{aligned}
2-k \alpha_{2}\left(\alpha_{1}^{-1}+\alpha_{2}^{-1}-1\right) & =1-\frac{\alpha_{2}}{\alpha_{1}}+\alpha_{2}\left(k-(k-1)\left(\alpha_{1}^{-1}+\alpha_{2}^{-1}\right)\right) \\
& \geq \alpha_{2}\left(k-(k-1)\left(\alpha_{1}^{-1}+\alpha_{2}^{-1}\right)\right)
\end{aligned}
$$

Hence the condition $k-(k-1)\left(\alpha_{1}^{-1}+\alpha_{2}^{-1}\right)>0$ is equivalent to $\operatorname{dim}_{\mathrm{H}} M_{k}>0$ a.s. When the stability exponent of $X$ satisfies the case (A.1), it follows from Theorem 2 that

$$
M_{k} \neq \emptyset \text { a.s. } \Longleftrightarrow \operatorname{dim}_{\mathrm{H}} M_{k}>0 \text { a.s. }
$$

However, as already mentioned in the Introduction, the last equivalence does not hold in the case (A.2), i.e., $X$ may have $k$-multiple points even if the Hausdorff dimension of $M_{k}$ is zero. An essential part of the proof is the following multiple integral estimate.

Proposition 5. Fix $k \in \mathbb{N}$ and assume $2(k-1) / k<\alpha<2$. Then for any real numbers $q, r \geq 3$ we have

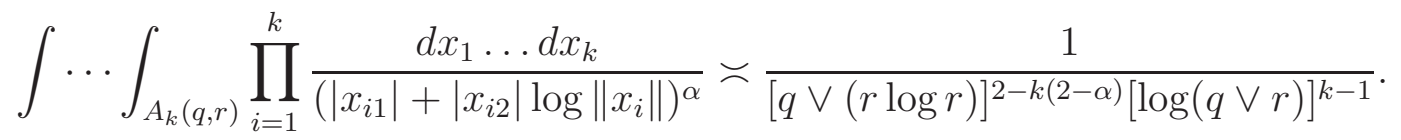


Proof. As in the proof of Proposition 3, we restrict the integration in (14) to the set

$$
\begin{gathered}
\widetilde{A}_{k}(q, r):=\left\{\left(x_{1}, \ldots, x_{k}\right) \in \mathbb{R}^{2 k}: x_{k 1}, x_{k 2} \geq 1,\left|x_{i 1}\right|,\left|x_{i 2}\right| \geq 1, i=1, \ldots, k-1,\right. \\
\left.q-1 \leq\left|\sum_{i=1}^{k} x_{i 1}\right|<q, r-1 \leq\left|\sum_{i=1}^{k} x_{i 2}\right|<r\right\},
\end{gathered}
$$

and we employ the sets $A_{k}^{i, j}(q, r)$ for $i, j=1,2,3,4$. Recall that

$$
A_{k}^{1,1}(q, r) \subseteq \widetilde{A}_{k}(q, r) \subseteq \bigcup_{i, j=1}^{4} A_{k}^{i, j}(q, r)
$$

so it is enough to show the lower bound of (14) with the integration restricted to $A_{k}^{1,1}(q, r)$ and the upper bound of (14) with the integration over all $A_{k}^{i, j}(q, r)$. We proceed by induction on $k$. The case $k=1$ is obvious, as

$$
\iint_{A_{1}(q, r)} \frac{d x_{1} d x_{2}}{\left(\left|x_{1}\right|+\left|x_{2}\right| \log \|x\|\right)^{\alpha}} \asymp[q+r \log (q+r)]^{-\alpha} \asymp[q \vee(r \log r)]^{-\alpha}
$$

Assume (14) holds for some $k \geq 1$ and set $k^{\prime}:=k+1$. Consider first the integration over $A_{k^{\prime}}^{1,1}(q, r)$. By the induction hypothesis applied to $A_{k}\left(q+x_{k^{\prime} 1}, r+x_{k^{\prime} 2}\right)$, we get

$$
\begin{aligned}
& \int \cdots \int_{A_{k^{\prime}(q, r)}^{1,1}} \prod_{i=1}^{k^{\prime}} \frac{d x_{1} \ldots d x_{k^{\prime}}}{\left(\left|x_{i 1}\right|+\left|x_{i 2}\right| \log \left\|x_{i}\right\|\right)^{\alpha}} \\
& \asymp \int_{1}^{\infty} \int_{1}^{\infty}\left(\frac{1}{q+x_{k^{\prime} 1}+\left(r+x_{k^{\prime} 2}\right) \log \left(r+x_{k^{\prime} 2}\right)}\right)^{2-k(2-\alpha)} \\
& \quad \times \frac{1}{\left(\frac{d x_{k^{\prime} 1} d x_{k^{\prime} 2}}{\log \left(q+x_{k^{\prime} 1}+r+x_{k^{\prime} 2}\right)}\right)^{k-1}} .
\end{aligned}
$$

We split the right-hand side into four integrals

$$
\begin{aligned}
\int_{1}^{\infty} \int_{1}^{\infty}(\ldots) & =\int_{1}^{r} \int_{1}^{q}(\ldots)+\int_{1}^{r} \int_{q}^{\infty}(\ldots)+\int_{r}^{\infty} \int_{1}^{q}(\ldots)+\int_{r}^{\infty} \int_{q}^{\infty}(\ldots) \\
& =I_{1}+I_{2}+I_{3}+I_{4} .
\end{aligned}
$$

Consider $I_{4}$ first. We have

$$
I_{4} \asymp \int_{r}^{\infty} \int_{q}^{\infty} \frac{d x d y}{(x+y \log y)^{2-k(2-\alpha)}[\log (x+y)]^{k-1}(x+y \log (x+y))^{\alpha}} .
$$


Assume $q<r \log r$. By Fubini,

$$
\begin{aligned}
I_{4} & \gtrsim \int_{r \log r}^{\infty} \int_{r}^{x} \frac{d y d x}{(x+y \log y)^{2-k(2-\alpha)}(\log x)^{k-1}(x+y \log x)^{\alpha}} \\
& \geq \int_{r \log r}^{\infty} \int_{r}^{x} \frac{d y d x}{(x+y \log x)^{k^{\prime} \alpha-2 k+2}(\log x)^{k-1}} \\
& =C \int_{r \log r}^{\infty} \frac{1}{(x+r \log x)^{k^{\prime} \alpha-2 k+1}(\log x)^{k}}\left[1-\left(\frac{x+r \log x}{x+x \log x}\right)^{k^{\prime} \alpha-2 k+1}\right] d x .
\end{aligned}
$$

Note that the condition $2\left(k^{\prime}-1\right) / k^{\prime}<\alpha$ implies $k^{\prime} \alpha-2 k>0$. Also, for $x \geq r \log r$ we have

$$
\frac{x+r \log x}{x+x \log x} \leq C<1
$$

Hence

$$
I_{4} \gtrsim \int_{r \log r}^{\infty} \frac{d x}{(x+r \log x)^{k^{\prime} \alpha-2 k+1}(\log x)^{k}} .
$$

Furthermore, since $x^{-1} \log x$ is decreasing, for $x \geq r \log r$ we get

$$
\frac{r \log x}{x} \leq \frac{r \log (r \log r)}{r \log r}=\frac{\log r+\log (\log r)}{\log r} \leq 2 .
$$

Therefore

$$
\begin{aligned}
& \int_{r \log r}^{\infty} \frac{d x}{(x+r \log x)^{k^{\prime} \alpha-2 k+1}(\log x)^{k}} \asymp \int_{r \log r}^{\infty} \frac{d x}{x^{k^{\prime} \alpha-2 k+1}(\log x)^{k}} \\
& \asymp \frac{1}{(r \log r)^{k^{\prime} \alpha-2 k}[\log (r \log r)]^{k}} \asymp \frac{1}{(r \log r)^{2-k^{\prime}(2-\alpha)}(\log r)^{k^{\prime}-1}} .
\end{aligned}
$$

Let $q \geq r \log r$. A similar estimate as above gives

$$
\begin{aligned}
I_{4} \gtrsim \int_{q}^{\infty} \int_{r}^{x} \frac{d y d x}{(x+y \log x)^{k^{\prime} \alpha-2 k+2}(\log x)^{k-1}} & \asymp \int_{q}^{\infty} \frac{d x}{(x+r \log x)^{k^{\prime} \alpha-2 k+1}(\log x)^{k}} \\
& \asymp \frac{1}{q^{2-k^{\prime}(2-\alpha)}(\log q)^{k^{\prime}-1}} .
\end{aligned}
$$

Altogether,

$$
I_{4} \gtrsim \frac{1}{[q \vee(r \log r)]^{2-k^{\prime}(2-\alpha)}[\log (q \vee r)]^{k^{\prime}-1}} .
$$

This also proves the lower bound of (14). It remains to show the upper bound. By (15) we have

$$
\begin{aligned}
I_{4} & \lesssim \int_{q}^{\infty} \int_{r}^{\infty} \frac{d y d x}{x^{2-k(2-\alpha)}(\log x)^{k-1}(x+y \log x)^{\alpha}} \\
& =C \int_{q}^{\infty} \frac{d x}{x^{2-k(2-\alpha)}(\log x)^{k}(x+r \log x)^{\alpha-1}} \\
& \lesssim \int_{q}^{\infty} \frac{d x}{x^{k^{\prime} \alpha-2 k+1}(\log x)^{k}} \asymp \frac{1}{q^{2-k^{\prime}(2-\alpha)}(\log q)^{k^{\prime}-1}}
\end{aligned}
$$


On the other hand,

$$
\begin{aligned}
I_{4} & \lesssim \int_{r}^{\infty} \int_{q}^{\infty} \frac{d x d y}{(x+y \log y)^{k^{\prime} \alpha-2 k+2}(\log y)^{k-1}}=C \int_{r}^{\infty} \frac{d y}{(q+y \log y)^{k^{\prime} \alpha-2 k+1}(\log y)^{k-1}} \\
& \lesssim \int_{r}^{\infty} \frac{d y}{y^{k^{\prime} \alpha-2 k+1}(\log y)^{k^{\prime} \alpha-k}} \asymp \frac{1}{r^{k^{\prime} \alpha-2 k}(\log r)^{k^{\prime} \alpha-k}}=\frac{1}{(r \log r)^{2-k^{\prime}(2-\alpha)}(\log r)^{k^{\prime}-1}} .
\end{aligned}
$$

By taking the minimum of two upper bounds in terms of $q$ and $r$ we get

$$
I_{4} \lesssim \frac{1}{[q \vee(r \log r)]^{2-k^{\prime}(2-\alpha)}[\log (q \vee r)]^{k^{\prime}-1}}
$$

Next we consider $I_{1}$. Notice that

$$
I_{1} \asymp \frac{1}{[q \vee(r \log r)]^{2-k(2-\alpha)}[\log (q \vee r)]^{k-1}} \int_{1}^{r} \int_{1}^{q} \frac{d x d y}{[x+y \log (x+y)]^{\alpha}} .
$$

Since $\alpha>1$, we get by Fubini's theorem

$$
\int_{1}^{r} \int_{1}^{q} \frac{d x d y}{[x+y \log (x+y)]^{\alpha}} \lesssim \int_{1}^{q} \int_{1}^{r} \frac{d y d x}{(x+y \log x)^{\alpha}} \lesssim \int_{1}^{q} \frac{d x}{x^{\alpha-1} \log x} \lesssim \frac{q^{2-\alpha}}{\log q} .
$$

Hence $I_{1} \lesssim q^{k^{\prime}(2-\alpha)-2}(\log q)^{1-k^{\prime}}$. In a similar manner,

$$
\int_{1}^{r} \int_{1}^{q} \frac{d x d y}{[x+y \log (x+y)]^{\alpha}} \lesssim \int_{1}^{r} \int_{1}^{q} \frac{d x d y}{(x+y \log y)^{\alpha}} \lesssim \int_{1}^{r} \frac{d y}{(y \log y)^{\alpha-1}} \lesssim \frac{r^{2-\alpha}}{(\log r)^{\alpha-1}} .
$$

This gives $I_{1} \lesssim(r \log r)^{k^{\prime}(2-\alpha)-2}(\log r)^{1-k^{\prime}}$, and the desired upper bound for $I_{1}$ follows. We have

$$
\begin{aligned}
I_{2} & \lesssim \int_{1}^{r} \int_{q}^{\infty} \frac{d x d y}{[x+(r \log r)]^{2-k(2-\alpha)}[\log (x+r)]^{k-1}[x+y \log (x+y)]^{\alpha}} \\
& \lesssim \frac{1}{(r \log r)^{2-k(2-\alpha)}(\log r)^{k-1}} \int_{1}^{r} \int_{q}^{\infty} \frac{d x d y}{[x+y \log (2 y)]^{\alpha}} \\
& \lesssim \frac{1}{(r \log r)^{2-k(2-\alpha)(\log r)^{k-1}}} \int_{1}^{r} \frac{d y}{(y \log (2 y))^{\alpha-1}} \\
& \lesssim \frac{1}{(r \log r)^{2-k(2-\alpha)}(\log r)^{k-1}} \cdot \frac{r^{2-\alpha}}{(\log r)^{\alpha-1}}=\frac{1}{(r \log r)^{2-k^{\prime}(2-\alpha)}(\log r)^{k^{\prime}-1}}
\end{aligned}
$$

Furthermore, by Fubini we obtain

$$
\begin{aligned}
I_{2} \leq \frac{1}{(\log q)^{k-1}} \int_{q}^{\infty} \int_{1}^{r} \frac{d y d x}{x^{2-k(2-\alpha)}(x+y \log x)^{\alpha}} & \lesssim \frac{1}{(\log q)^{k-1}} \int_{q}^{\infty} \frac{d x}{x^{\alpha-k(2-\alpha)+1} \log x} \\
& \asymp \frac{1}{q^{2-k^{\prime}(2-\alpha)}(\log q)^{k^{\prime}-1}} .
\end{aligned}
$$

This gives the upper bound for $I_{2}$. Estimating $I_{3}$ is similar. Therefore we have proved the upper bound for the integral in (14) over $A_{k^{\prime}}^{1,1}(q, r)$.

Consider the integration over $A_{k^{\prime}}^{2,2}(q, r)$. Note that we need to modify slightly the definition of the latter as the argument of the logarithm on the left-hand side of (14) 
does not approach 1 . By the induction hypothesis applied to $A_{k}\left(q-x_{k^{\prime} 1}, r-x_{k^{\prime} 2}\right)$ we have

$$
\begin{aligned}
\int \cdots \int_{A_{k^{\prime}(q, r)}^{2,2}} \prod_{i=1}^{k^{\prime}} \frac{d x_{1} \ldots d x_{k^{\prime}}}{\left(\left|x_{i 1}\right|+\left|x_{i 2}\right| \log \| x_{i}||\right)^{\alpha}} & \\
\asymp \int_{3}^{r-3} \int_{3}^{q-3}\left(\frac{1}{q-x+(r-y) \log (r-y)}\right)^{2-k(2-\alpha)}\left(\frac{1}{\log (q-x+r-y)}\right)^{k-1} & \times \frac{d x d y}{[x+y \log (x+y)]^{\alpha}} .
\end{aligned}
$$

As in the previous case, we split the right-hand side into

$$
\begin{aligned}
& \int_{3}^{r / 2} \int_{3}^{q / 2}(\ldots)+\int_{r / 2}^{r-3} \int_{q / 2}^{q-3}(\ldots)+\int_{3}^{r / 2} \int_{q / 2}^{q-3}(\ldots)+\int_{r / 2}^{r-3} \int_{3}^{q / 2}(\ldots) \\
& =J_{1}+J_{2}+J_{3}+J_{4} .
\end{aligned}
$$

We have

$$
J_{1} \asymp \frac{1}{[q \vee(r \log r)]^{2-k(2-\alpha)}[\log (q \vee r)]^{k-1}} \int_{3}^{r / 2} \int_{3}^{q / 2} \frac{d x d y}{[x+y \log (x+y)]^{\alpha}} \asymp I_{1},
$$

and the desired upper bound follows from the estimate of $I_{1}$. Furthermore,

$$
J_{2} \asymp \frac{1}{[q \vee(r \log r)]^{\alpha}} \int_{3}^{r / 2} \int_{3}^{q / 2} \frac{d x d y}{(x+y \log y)^{2-k(2-\alpha)}[\log (x+y)]^{k-1}} .
$$

As $\alpha=2-k(2-\alpha)+(k-1)(2-\alpha)$, we get

$$
J_{2} \lesssim \frac{1}{[q \vee(r \log r)]^{2-k(2-\alpha)}} \int_{3}^{r / 2} \int_{3}^{q / 2} \frac{d x d y}{(x+y \log y)^{\alpha}[\log (x+y)]^{k-1}} .
$$

Note that $x+y \log y \asymp x+y \log (x+y)$. Using similar arguments as for $I_{1}$ we get

$$
\int_{3}^{r / 2} \int_{3}^{q / 2} \frac{d x d y}{[x+y \log (x+y)]^{\alpha}[\log (x+y)]^{k-1}} \lesssim \frac{q^{2-\alpha}}{(\log q)^{k}} \wedge \frac{r^{2-\alpha}}{(\log r)^{\alpha+k-2}} .
$$

This gives the upper bound for $J_{2}$. Since the cases of $J_{3}$ and $J_{4}$ are very similar to each other, we consider only the first one. For the latter one gets

$$
\begin{aligned}
J_{3} & \asymp \int_{3}^{r / 2} \int_{q / 2}^{q-3} \frac{d x d y}{(q-x+r \log r)^{2-k(2-\alpha)}[\log (q-x+r)]^{k-1}[x+y \log (x+y)]^{\alpha}} \\
& =\int_{3}^{r / 2} \int_{3}^{q / 2} \frac{d x d y}{(x+r \log r)^{2-k(2-\alpha)}[\log (x+r)]^{k-1}[q-x+y \log (q-x+y)]^{\alpha}} \\
& \asymp \int_{3}^{r / 2} \int_{3}^{q / 2} \frac{d x d y}{(x+r \log r)^{2-k(2-\alpha)}[\log (x+r)]^{k-1}[q+y \log (q+y)]^{\alpha}} .
\end{aligned}
$$

Hence we have

$$
J_{3} \lesssim \frac{1}{(r \log r)^{2-k(2-\alpha)}(\log r)^{k-1}} \int_{3}^{r / 2} \int_{3}^{q / 2} \frac{d x d y}{[x+y \log (x+y)]^{\alpha}},
$$


and applying the previous estimate for $I_{1}$ we get the desired upper bound in terms of $r$. In order to estimate $J_{3}$ in terms of $q$ we can assume $q \geq r \log r$. We write

$$
J_{3} \asymp \int_{3}^{r / 2} \int_{3}^{q / 2} \frac{(x+r \log r)^{(k-1)(2-\alpha)} d x d y}{(x+r \log r)^{\alpha}[\log (x+r)]^{k-1}[q+y \log (q+y)]^{\alpha}} .
$$

As $q+y \log (q+y) \asymp q$ for $y \leq r$ we get

$$
\begin{aligned}
J_{3} & \lesssim q^{k(2-\alpha)-2} \int_{3}^{r / 2} \int_{3}^{q / 2} \frac{d x d y}{(x+r \log r)^{\alpha}(\log x)^{k-1}} \\
& \lesssim q^{k(2-\alpha)-2} \int_{3}^{r / 2} \int_{3}^{q / 2} \frac{d x d y}{[x+y \log (x+y)]^{\alpha}(\log x)^{k-1}},
\end{aligned}
$$

and since the last integral has already appeared in the case of $J_{2}$, the upper bound for $J_{3}$ is proved. This also completes the upper estimate with integration over $A_{k^{\prime}}^{2,2}(q, r)$. The remaining integrals can be estimated in a similar way or reduced to the cases already considered by splitting the domain of integration in a suitable way. We omit the details.

Proof of Theorem 2, Case (A.2). As in the proof for the case (A.1), we may assume without loss of generality that $\alpha<2$. By [14, (2.1),(2.5),(2.6),(2.7)], we have

$$
\Psi(x) \asymp\left|x_{1}\right|^{\alpha}+\left|x_{2}\right|^{\alpha}(\ln \|x\|)^{\alpha} \quad \text { for }\|x\| \geq C,
$$

with $\alpha:=\alpha_{1}=\alpha_{2}=1 / a$. In order to apply this estimate to (12) we split the domain of integration into $\left\|\sum_{j=1}^{k-1} x_{j}\right\| \leq 2$ and $\left\|\sum_{j=1}^{k-1} x_{j}\right\| \geq 2$. Note that the condition $\left\|\sum_{j=1}^{k-1} x_{j}\right\| \leq 2$ implies

$$
\left|x_{(k-1) 1}\right| \asymp\left|\sum_{j=1}^{k-2} x_{j 1}\right| \quad \text { and } \quad\left|x_{(k-1) 2}\right| \asymp\left|\sum_{j=1}^{k-2} x_{j 2}\right| \quad \text { for }\left\|x_{k-1}\right\| \geq C .
$$

This means that

$$
\int_{\mathbb{R}^{d(k-1)}} \frac{1}{1+\Psi\left(\sum_{j=1}^{k-1} x_{j}\right)} \mathbb{1}_{\left\{\left\|\sum_{j=1}^{k-1} x_{j}\right\| \leq 2\right\}} \prod_{j=1}^{k-1} \frac{1}{1+\Psi\left(x_{j}\right)} d \bar{x}<\infty
$$

if and only if

$$
\int_{\mathbb{R}^{d(k-2)}} \frac{1}{1+\Psi\left(\sum_{j=1}^{k-2} x_{j}\right)} \prod_{j=1}^{k-2} \frac{1}{1+\Psi\left(x_{j}\right)} d \bar{x}<\infty .
$$

Since the last condition is equivalent to $M_{k-1} \neq \emptyset$, it is enough to consider the case $\left\|\sum_{j=1}^{k-1} x_{j}\right\| \geq 2$, and the final conclusion will follow by induction. The estimates of $\Psi$ imply that

$$
\int_{\mathbb{R}^{d(k-1)}} \frac{1}{1+\Psi\left(\sum_{j=1}^{k-1} x_{j}\right)} \mathbb{1}_{\left\{\left\|\sum_{j=1}^{k-1} x_{j}\right\| \geq 2\right\}} \prod_{j=1}^{k-1} \frac{1}{1+\Psi\left(x_{j}\right)} d \bar{x}<\infty
$$


if and only if

$$
\begin{aligned}
\int_{\mathbb{R}^{2(k-1)}} & \frac{1}{1+\left|\sum_{j=1}^{k-1} x_{j 1}\right|^{\alpha}+\left|\sum_{j=1}^{k-1} x_{j 2}\right|^{\alpha}\left(\log \left\|\sum_{j=1}^{k-1} x_{j}\right\|\right)^{\alpha}} \\
& \times \mathbb{1}_{\left\{\left\|\sum_{j=1}^{k-1} x_{j}\right\| \geq 2\right\}} \prod_{j=1}^{k-1} \frac{1}{1+\left|x_{j 1}\right|^{\alpha}+\left|x_{j 2}\right|^{\alpha}\left(\log \left\|x_{j}\right\|\right)^{\alpha}} d \bar{x}<\infty .
\end{aligned}
$$

Employing the sets $A_{k-1}(m, n)$ as in the proof for the case (A.1), we conclude that the last condition is equivalent to

$$
\sum_{m, n \geq 3}\left(\frac{1}{m+n \log (m+n)}\right)^{\alpha} \int \cdots \int_{A_{k-1}(m, n)} \prod_{i=1}^{k-1} \frac{d x_{1} \ldots d x_{k-1}}{\left(\left|x_{i 1}\right|+\left|x_{i 2}\right| \log \left\|x_{i}\right\|\right)^{\alpha}}<\infty .
$$

Finally, Proposition 5 with $k-1$ instead of $k$ implies that (16) holds if and only if

$$
\sum_{m, n \geq 3}\left(\frac{1}{m+n \log (m+n)}\right)^{\alpha} \frac{1}{[m \vee(n \log n)]^{2-(k-1)(2-\alpha)}[\log (m \vee n)]^{k-2}}<\infty
$$

As already argued in the proof of Proposition 5, we have $m+n \log (m+n) \asymp m \vee$ $(n \log n)$. Split the series in (17) into two parts

$$
\sum_{m, n \geq 3}(\ldots)=\sum_{m \leq n \log n}(\ldots)+\sum_{m>n \log n}(\ldots)=S_{1}+S_{2}
$$

We get

$$
S_{1} \asymp \sum_{m \leq n \log n} \frac{1}{n^{k \alpha-2 k+4}(\log n)^{k \alpha-k+2}} \asymp \sum_{n \geq 3} \frac{1}{n^{k \alpha-2 k+3}(\log n)^{k \alpha-k+1}} .
$$

Thus $S_{1}<\infty$ if and only if $\alpha \geq 2(k-1) / k$. Furthermore,

$$
S_{2} \leq \sum_{m>n \log n} \frac{1}{m^{k \alpha-2 k+4}(\log m)^{k-2}}
$$

For $\alpha \geq 2(k-1) / k$ we have

$$
\begin{aligned}
\int_{3}^{\infty} \int_{x \log x}^{\infty} \frac{d y d x}{y^{k \alpha-2 k+4}(\log y)^{k-2}} & \asymp \int_{3}^{\infty} \frac{d x}{(x \log x)^{k \alpha-2 k+3}[\log (x \log x)]^{k-2}} \\
& \asymp \int_{3}^{\infty} \frac{d x}{x^{k \alpha-2 k+3}(\log x)^{k \alpha-k+1}}<\infty,
\end{aligned}
$$

and so $S_{2}<\infty$. Hence $S_{1}+S_{2}<\infty$ if and only if $\alpha \geq 2(k-1) / k$, as desired.

We finish this section by characterizing the existence of double points of $X$. According to the Jordan decomposition for $d=3$, the stability exponent of $X$ satisfies $B=P D P^{-1}$, where the matrix $D$ may have one of the the following forms: 


$$
\begin{aligned}
& \left(\begin{array}{ccc}
1 / \alpha_{1} & 0 & 0 \\
0 & 1 / \alpha_{2} & 0 \\
0 & 0 & 1 / \alpha_{3}
\end{array}\right),\left(\begin{array}{ccc}
1 / \alpha_{1} & -b & 0 \\
b & 1 / \alpha_{2} & 0 \\
0 & 0 & 1 / \alpha_{3}
\end{array}\right) \text { with } \alpha_{1}=\alpha_{2} \\
& \text { or }\left(\begin{array}{ccc}
1 / \alpha_{1} & 0 & 0 \\
0 & 1 / \alpha_{2} & -b \\
0 & b & 1 / \alpha_{3}
\end{array}\right) \text { with } \alpha_{2}=\alpha_{3}
\end{aligned}
$$

(B.2) $\left(\begin{array}{ccc}1 / \alpha_{1} & 0 & 0 \\ 1 & 1 / \alpha_{2} & 0 \\ 0 & 0 & 1 / \alpha_{3}\end{array}\right)$ with $\alpha_{1}=\alpha_{2}$ or $\left(\begin{array}{ccc}1 / \alpha_{1} & 0 & 0 \\ 0 & 1 / \alpha_{2} & 0 \\ 0 & 1 & 1 / \alpha_{3}\end{array}\right)$ with $\alpha_{2}=\alpha_{3}$;

(B.3) $\left(\begin{array}{ccc}1 / \alpha & 0 & 0 \\ 1 & 1 / \alpha & 0 \\ 0 & 1 & 1 / \alpha\end{array}\right)$

Clearly, in the case (B.3) we have $\alpha:=\alpha_{1}=\alpha_{2}=\alpha_{3}$.

Corollary 6. Let $X=\left\{X(t), t \in \mathbb{R}_{+}\right\}$be a symmetric operator semistable Lévy process in $\mathbb{R}^{d}$. A necessary and sufficient condition for the existence of double points of $X$ is the following:

- $d=2: M_{2} \neq \emptyset$ almost surely if and only if $2-1 / \alpha_{1}-1 / \alpha_{2}>0$.

- $d=3$, Cases (B.1) and (B.2): $M_{2} \neq \emptyset$ almost surely if and only if $2-1 / \alpha_{1}-$ $1 / \alpha_{2}-1 / \alpha_{3}>0$.

- $d=3$, Case (B.3): $M_{2} \neq \emptyset$ almost surely if and only if $\alpha \geq 3 / 2$.

Furthermore, $M_{2}=\emptyset$ almost surely for $d \geq 4$.

Proof. The corollary is a reformulation of [23, Theorem 5 and Theorem 7], where the result was proved for symmetric operator stable Lévy processes. The proof was based on (12) and on the asymptotics of the characteristic exponent derived in [26, (4.9),(4.14),(4.15),(4.16)]. Analogous asymptotics for operator semistable Lévy processes were obtained in [14, (2.1),(2.5),(2.6),(2.7)]. Therefore, the proof is the same as in 23. The last statement is justified at the beginning of this section.

Remark 7. We note that the classification of the stability exponent $B$ and the corresponding asymptotics of the Lévy exponent at infinity in [23, Proofs of Theorems 5 and 7] was not quite correct. In dimension 2 and $B=P D P^{-1}$ with

$$
D=\left(\begin{array}{cc}
1 / \alpha_{1} & -b \\
b & 1 / \alpha_{2}
\end{array}\right) \text { and } \alpha_{1}=\alpha_{2},
$$

we remark that since the matrix is diagonalizable over the complex numbers, it follows from [14, $(2.1),(2.5),(2.6),(2.7)]$ that

$$
\Psi(x) \asymp\left|x_{1}\right|^{\alpha_{1}}+\left|x_{2}\right|^{\alpha_{2}} \quad \text { for }\|x\| \geq C .
$$


Similarly, in dimension 3 and $B=P D P^{-1}$ with

$$
D=\left(\begin{array}{ccc}
1 / \alpha_{1} & -b & 0 \\
b & 1 / \alpha_{2} & 0 \\
0 & 0 & 1 / \alpha_{3}
\end{array}\right) \quad \text { and } \alpha_{1}=\alpha_{2}
$$

or

$$
D=\left(\begin{array}{ccc}
1 / \alpha_{1} & 0 & 0 \\
0 & 1 / \alpha_{2} & -b \\
0 & b & 1 / \alpha_{3}
\end{array}\right) \quad \text { and } \alpha_{2}=\alpha_{3},
$$

it follows from [14, (2.1),(2.5),(2.6),(2.7)] that

$$
\Psi(x) \asymp\left|x_{1}\right|^{\alpha_{1}}+\left|x_{2}\right|^{\alpha_{2}}+\left|x_{3}\right|^{\alpha_{3}} \quad \text { for }\|x\| \geq C .
$$

This, however, does not change the statements of [23, Theorems 5 and 7].

For completeness, we end this paper with the following remark on multiple points of a symmetric semistable Lévy process in $\mathbb{R}$.

Remark 8. The existence and Hausdorff dimension of multiple points for symmetric stable Lévy processes in $\mathbb{R}$ have been fully characterized in [29]. If $X$ is a real-valued symmetric semistable Lévy process with characteristic exponent $\Psi(\xi)$, the existence of multiple points and the Hausdorff dimension of $M_{k}$ can be explicitly determined by applying (12) and (3), which only depend on the asymptotic behavior of $\Psi(\xi)$ at $\infty$. It follows from Choi [2, Remark 2] that the characteristic exponent of $X$ is of the form $\Psi(\xi)=|\xi|^{\alpha} R(\xi)$, where $0<\alpha<2$ is a constant and $R(\xi)$ is a non-negative bounded and continuous function on $\mathbb{R}$. Hence, if

$$
\inf \{R(\xi):|\xi| \geq 1\}>0
$$

(by [2, Remark 2] this holds if the support of the Lévy measure of $X$ spans $\mathbb{R}$ ), then $\Psi(\xi) \asymp|\xi|^{\alpha}$ for $\xi \in \mathbb{R}$ with $|\xi| \geq 1$. In view of the formula (3) and the condition (12), we can verify that under (18) the characterizations of multiple points of $X$ are the same as those in [29, Theorems 1 and 3] for a symmetric $\alpha$-stable Lévy process in $\mathbb{R}$.

\section{REFERENCES}

[1] J. Bertoin, Lévy Processes, Cambridge Tracts in Mathematics, Cambridge, 1996.

[2] G.S. Choi, Criteria for recurrence and transience of semistable processes, Nagoya Math. J. 134 (1994), 91-106.

[3] V. Chorny, Operator semistable distributions on $\mathbb{R}^{d}$, Theory Probab. Appl. 31 (1987), 703-709.

[4] A. Dvoretzky, P. Erdös, S. Kakutani, Double points of paths of Brownian motion in n-space, Acta Sci. Math. 12 (1950), 75-81.

[5] A. Dvoretzky, P. Erdös, S. Kakutani, Multiple points of paths of Brownian motion in the plane, Bull. Res. Council Israel, Sect. F 3 (1954), 364-371.

[6] A. Dvoretzky, P. Erdös, S. Kakutani, S.J. Taylor, Triple points of Brownian motion in 3-space, Proc. Cambridge Philos. Soc. 53 (1957), 856-862.

[7] E.B. Dynkin, Random fields associated with multiple points of the Brownian motion, J. Funct. Anal. 62 (1985), 397-434.

[8] S.N. Evans, Multiple points in the sample paths of a Lévy process, Probab. Theory Relat. Fields 76 (1987), 359-367. 
[9] B. Fristedt, An extension of a theorem of S. J. Taylor concerning the multiple points of the symmetric stable process, Z. Wahrsch. Verw. Gebiete 9 (1967), 62-64.

[10] P.J. Fitzsimmons, T.S. Salisbury, Capacity and energy for multiparameter Markov processes, Ann. Inst. H. Poincaré Probab. Statist. 25 (1989) 325-350.

[11] J. Hawkes, Multiple points for symmetric Lévy processes, Math. Proc. Cambridge Philos. Soc. 83 (1978), 83-90.

[12] W.J. Hendricks, Multiple points for transient symmetric Lévy processes, Z. Wahrsch. Verw. Gebiete 49 (1979), 13-21.

[13] R. Jajte, Semi-stable probability measures on $\mathbb{R}^{N}$, Studia Math. 61 (1977), 29-39.

[14] P. Kern, M.M. Meerschaert, Y. Xiao, Asymptotic behavior of semistable Lévy exponents and applications to fractal path properties, J. Theoret. Probab. 31 (2018), 598-617.

[15] D. Khoshnevisan, Intersections of Brownian motions, Expo. Math. 21 (2003), 97-114.

[16] D. Khoshnevisan, Y. Xiao, Level sets of additive Lévy processes, Ann. Probab. 30 (2002), 62-100.

[17] D. Khoshnevisan, Y. Xiao, Weak unimodality of finite measures, and an application to potential theory of additive Lévy processes, Proc. Amer. Math. Soc., 131 (2003), 2611-2616.

[18] D. Khoshnevisan, Y. Xiao, Additive Lévy processes: capacity and Hausdorff dimension, In: Proc. of Inter. Conf. of Fractal Geometry and Stochastics III., Progr. Probab. 57 (2004), 62-100.

[19] D. Khoshnevisan, Y. Xiao, Harmonic analysis of additive Lévy processes, Probab. Theory Relat. Fields 145 (2009), 459-515.

[20] R.G. Laha, V.K. Rohatgi, Operator semistable probability measures on a Hilbert space, Bull. Austral. Math. Soc. 22 (1980), 397-406.

[21] J.-F. Le Gall, J.S. Rosen, N.-R. Shieh, Multiple points of Lévy processes, Ann. Probab. 17 (1989), $503-515$.

[22] A. Łuczak, Operator semi-stable probability measures on $\mathbb{R}^{N}$, Coll. Math. 45 (1981), 287-300.

[23] T. Luks, Y. Xiao, On the double points of operator stable Lévy processes, J. Theoret. Probab. 30 (2017), no. 1, 297-325.

[24] M. Maejima, K. Sato, Semi-selfsimilar processes, J. Theoret. Probab. 12 (1999), 347-373.

[25] M.M. Meerschaert, H.-P. Scheffler, Limit Distributions for Sums of Independent Random Vectors, John Wiley, New York, 2001.

[26] M.M. Meerschaert, Y. Xiao, Dimension results for sample paths of operator stable Lévy processes, Stochastic Process. Appl. 115 (2005), 55-75.

[27] K. Sato, Lévy Processes and Infinitely Divisible Distributions, Cambridge Univ. Press, Cambridge, 1999.

[28] N.-R. Shieh, Multiple points of dilation-stable Lévy processes, Ann. Probab. 26 (1998), 13411355.

[29] S.J. Taylor, Multiple points for the sample paths of the symmetric stable processes, Z. Wahrsch. Verw. Geb. 5 (1966), 247-264.

[30] Y. Xiao, Random fractals and Markov processes, In: Fractal Geometry and Applications: A Jubilee of Benoit Mandelbrot, (Michel L. Lapidus and Machiel van Frankenhuijsen, editors), pp. 261-338, American Mathematical Society, 2004.

Institut für Mathematik, Universität PAderborn, Warburger Strasse 100, D-33098 PADERBorn, Germany

E-mail address: tluks@math.uni-paderborn.de

Department of Statistics and Probability, Michigan State University, 619 Red Cedar Road, C413 Wells Hall, East Lansing MI 48824-1027, USA

E-mail address: xiaoyimi@stt.msu.edu 